\title{
On Bead Peptoid Dimerization Induced by Incorporation of Glycosylated Bridging Units in Submonomer Solid-Phase Approach to Glycopeptoids
}

Daniela Comegna, Annarita Del Gatto, Michele Saviano and Laura Zaccaro

Institute of Biostructures and Bioimaging-CNR, Via Mezzocannone 16, 80134 Naples, Italy; Interdepartmental Center of Bioactive Peptide, University of Naples Federico II, Via Mezzocannone 16, 80134 Naples, Italy; Institute of Crystallography-CNR, Via Amendola 122/O, 70126 Bari, Italy

daniela.comegna@unina.it; lzaccaro@unina.it

SUPPORTING INFORMATION

Table of content

$\begin{array}{ll}\text { List of Abbreviation } & \text { S2 }\end{array}$

$\begin{array}{ll}\text { General procedures } & \mathrm{S} 3\end{array}$

Solid-Phase Synthesis of Glycosylated Peptoids and Dimers $\quad$ S4

$\begin{array}{ll}\text { Purity Analysis } & \text { S5 }\end{array}$

Peptoid Purification $\quad$ S5

$\begin{array}{ll}\text { Calibration Curve } & \text { S5 }\end{array}$

$\begin{array}{ll}\text { Characterization of Compounds } & \text { S7 }\end{array}$

$\begin{array}{ll}\text { NMR spectra } & \text { S11 }\end{array}$

LC-MS Analysis and Integration Peak List $\quad$ S16 


\section{LIST OF ABBREVIATIONS}

$\mathrm{ACN}$

DCM

DMF

DIPEA

$\mathrm{MeOH}$

TEA

TFA

HIFP
Acetonitrile

Dichloromethane

$N, N$-dimethylformamide

$N, N$-diisopropylethylamine

Methanol

Triethylamine

Trifluoroacetic acid

Hexafluro-2-propanol 


\section{General procedures}

${ }^{1} \mathrm{H}$ and ${ }^{13} \mathrm{C}$ NMR spectra were recorded on Varian Unity Inova 400 NMR spectrometer equipped with dual probe $(400.13 \mathrm{MHz}$ for $1 \mathrm{H}, 100.13 \mathrm{MHz}$ for $13 \mathrm{C})$. Chemical shifts $(\delta)$ are reported in ppm relatively to the residual solvent peak $\left(\mathrm{CHCl}_{3}, \delta=7.26,{ }^{13} \mathrm{CDCl}_{3}, \delta:=77.0\right)$. High resolution ESI-MS spectra and analytical RP-HPLC analyses were performed on AGILENT Q-TOF LC/MS instrument equipped with DUAL ESI source and diode array detector using Phenomenex Aeris Peptides C18 column $(3.6 \mu \mathrm{m}, 2.1 \mathrm{~mm} \times 50 \mathrm{~mm})$.

For all RP-HPLC procedures, the system solvent was: $\mathrm{H}_{2} \mathrm{O} 0.1 \%$ TFA (A) and ACN 0.1\% TFA (B). Peptide purification was performed by RP-HPLC using a Phenomenex Jupiter Proteo C12 column $(10 \mu \mathrm{m}, 250 \mathrm{~mm} \times 10.00 \mathrm{~mm})$ with a flow rate of $10 \mathrm{~mL} \mathrm{~min}^{-1}$ and a linear gradient starting from $5 \%$ to $70 \% \mathrm{~B}$ in $40 \mathrm{~min}$.

Analytical analysis was performed on an Agilent 1200 system equipped with a Diode array detector with a flow rate of $300 \mu \mathrm{L} \mathrm{min}$ m $^{-1}$ using a linear gradient starting $5 \%$ to $70 \% \mathrm{~B}$ in $12 \mathrm{~min}$.

Analytical thin layer chromatography (TLC) was performed on aluminum plates precoated with Merck Silica Gel $60 \mathrm{~F}_{254}$ as the adsorbent. The plates were developed with $5 \% \mathrm{H}_{2} \mathrm{SO}_{4}$ ethanolic solution and then heating to $130^{\circ} \mathrm{C}$. Column chromatography was performed on Merck Kieselgel 60 (63-200 mesh). All reactions were carried out using dry solvents stored on molecular sieves.

2-Chlorotrytil chloride directly grafted on polystyrene (2, $\alpha$-dichloro benzhydryl-polystyrene crosslinked with 1\% DVB; 100-200 mesh; $1.6 \mathrm{mmol} / \mathrm{g}$ ), Novasyn TGT alcohol PEG-PS based resin (loading $0.16 \mathrm{mmol} / \mathrm{g}$ ), Novasyn TGR PEG-PS copolymer (loading $0.25 \mathrm{mmol} / \mathrm{g}$ ) were purchased from Novabiochem-Merck, all remaining solvents and reagents were purchased from Aldrich or Fluka) and were used without further purification. 


\section{Solid-Phase Synthesis of Glycosylated Peptoids and Dimers}

Linear peptoid oligomers were synthesized by using a submonomer solid-phase approach ${ }^{1}$.

All glycosylated peptoids and their corresponding dimers were synthesized on 2-chlorotrityl chloride resin (2, $\alpha$-dichloro benzhydryl-polystyrene crosslinked with 1\% DVB; 100-200 mesh) using different loading rates. To obtain the monomer as the main product low loading rates were used (from $0.1 \mathrm{up}$ to $1.0 \mathrm{mmol} / \mathrm{g}$ ) depending on the peptoid sequence, whereas to increase the dimer formation a loading of $1.6 \mathrm{mmol} / \mathrm{g}$ was used.

2-chlorotrityl chloride resin was swelled in dry DMF for 45 min and washed twice with dry DCM. The first sub-monomer was attached to the resin by adding bromoacetic acid ( 2 eq. relative to the resin) and DIPEA (4 eq. relative to carboxylic acid) in dry DCM and the mixture was stirred for 2 hours on a shaker platform for $30 \mathrm{~min}$ at room temperature, followed by washing with dry DCM and then with DMF for three times). The bromoacetylated resin was treated with a $1 \mathrm{M}$ DMF solution of the desired amine for $30 \mathrm{~min}$ at room temperature, then the resin was washed with DMF three times. Subsequent bromoacetylation reactions were accomplished by reacting 1.2M DMF solution of bromoacetic acid and DIC (1.1 eq. relative to bromoacetic acid) for $20 \mathrm{~min}$ at room temperature. The filtered resin was washed with DMF and treated again with the amine. This cycle of reactions was iterated until the obtainment of the target oligomer. The glycosylated moiety was obtained by adding the desired thioglycosylated amine ${ }^{2}$ and then the resin was cleaved to obtain a mixture of the glycosylpeptoid and its corresponding dimer. The cleavage was performed by treating twice the resin, previously washed with DCM, with of HFIP 20\% DCM solution in (v/v) on a shaker platform at room temperature for $30 \mathrm{~min}$ and 5 min respectively. The resin was then filtered away and the combined filtrates were concentrated in vacuo.

Glycosylpeptoid 5 was synthesized on Novasyn TGT alcohol PEG-PS based resin (loading 0.16 $\mathrm{mmol} / \mathrm{g}$ ). The resin was placed in a sintered glass funnel and washed with DMF, dry DCM and dry toluene. Then the resin was drained and transferred to a round bottom flask equipped with a reflux condenser. Toluene was added to cover the resin and then dropwise the acetyl chloride $(1 \mathrm{ml} / \mathrm{g}$ of resin). The mixture was heated at $70^{\circ} \mathrm{C}$ for 3 hours and then transferred to a sintered glass funnel and the resin washed with dry toluene and dry DCM, then drained. Bromoacetic acid (2 eq. relative to the resin) and DIPEA (4 eq. relative to carboxylic acid) in dry DCM were added to the resin and the mixture was stirred for 2 hours. The synthesis was carried out by using common submonomer solid-

\footnotetext{
${ }^{1}$ Zuckermann, N. R.; Kerr, J. M.; Kent, S. B. H. and Moos, W. H. J. Am. Chem. Soc. 1992, 114, 10646-10647.

${ }^{2}$ Comegna, D.; De Riccardis, F. Org. Lett., 2009, 11, 3898-3901.
} 
phase approach as described before. The cleavage was performed by treating twice the resin, previously washed with DCM, with of HFIP $20 \%$ in DCM (v/v) on a shaker platform at room temperature for $30 \mathrm{~min}$ and $5 \mathrm{~min}$ respectively. The resin was then filtered away and the combined filtrates were concentrated in vacuo.

\section{Purity Analysis}

The final products were analysed by HPLC and purities were determined by integration at $210 \mathrm{~nm}$. A C18 column (Waters, $\mu$ Bondapak, $10 \mu \mathrm{m}, 125 \AA 3.9 \mathrm{~mm} \times 300 \mathrm{~mm}$ ) was used for analysis of all peptoid products. The gradient elution was $5-100 \%$ acetonitrile/water $(0.1 \%$ TFA) in 30 minutes. Fractions containing the desired products were collected and analyzed by ESI-MS spectrometry.

\section{Peptoid Purification}

Peptoids were purified by reverse-phase HPLC using a C18 column (Waters, $\mu$ Bondapak, $10 \mu \mathrm{m}$, $125 \AA 7.8 \times 300 \mathrm{~mm})$. Gradient elution was 5-50\% acetonitrile/water $(0.1 \%$ TFA) in 30 minutes.

\section{Calibration Curve}

The standard curve was obtained using different concentrations of monomeric $(\mathbf{1}, \mathbf{3})$ and dimeric $(2$, 4) compounds, i.e., $0.1 \mathrm{mM}, 0.2 \mathrm{mM}, 0.3 \mathrm{mM}, 0.4 \mathrm{mM}$ and $0.5 \mathrm{mM}$. For each standard solution 20 $\mu \mathrm{l}$ were injected at LC-MS analysis system and the area under the curves of the integrated chromatogram were calculated. Each experiment was repeated three times.

\begin{tabular}{|c|c|c|}
\hline $\mathrm{C}(\mathrm{mM})$ & Area & Compound 1 \\
\hline 0.2 & 3214.13 & \\
\hline 0.4 & 6156.92 & \\
\hline 0.5 & 8452.40 & \\
\hline 0.2 & 6149.22 & Compound $\mathbf{2}$ \\
\hline 0.4 & 13460.35 & \\
\hline 0.5 & 16372.45 & \\
\hline 0.2 & 11893.18 & Compound $\mathbf{3}$ \\
\hline 0.4 & 23344.42 & \\
\hline 0.5 & 29019.64 & \\
\hline 0.1 & 12838.91 & Compound $\mathbf{4}$ \\
\hline 0.2 & 25499.55 & \\
\hline 0.3 & 36242.12 & \\
\hline
\end{tabular}




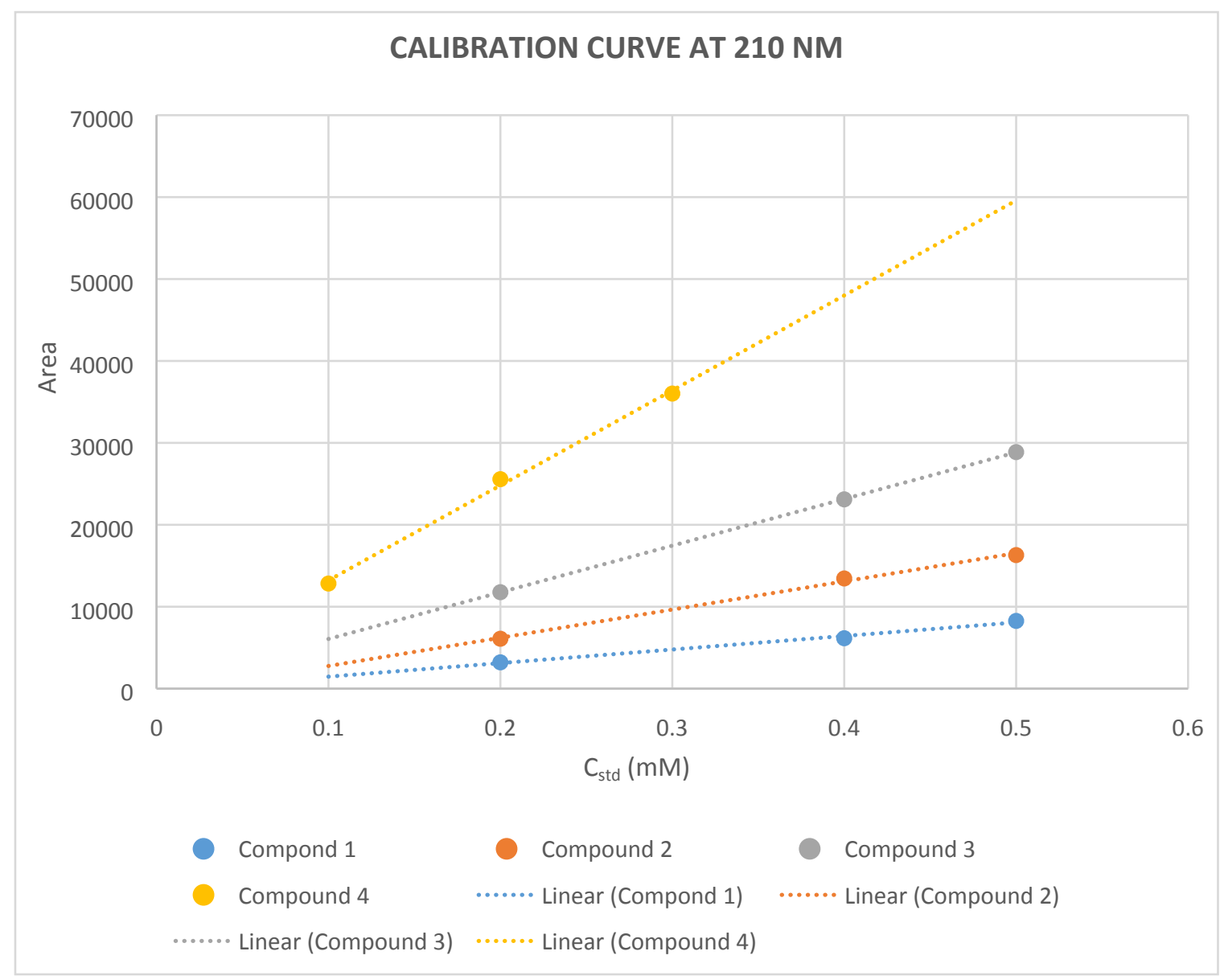




\section{Characterization of Compounds}

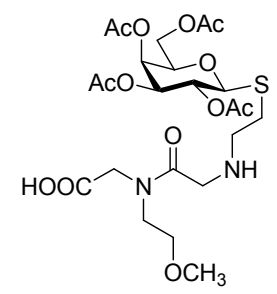

(1): ${ }^{1} \mathrm{H} \mathrm{NMR}\left(\mathrm{CDCl}_{3}, 400 \mathrm{MHz}\right.$, mixture of rotamers) $\delta 5.45\left(\mathrm{~d}, 1 \mathrm{H}, J_{4,3}=3.0 \mathrm{~Hz}, \mathrm{H}-4\right), 5.20(\mathrm{t}, 1 \mathrm{H}$, $\left.J_{2,3}=J_{2,1}=10.0 \mathrm{~Hz}, \mathrm{H}-2\right), 5.08\left(\mathrm{dd}, 1 \mathrm{H}, J_{3,2}=10.0, J_{3,4}=3.0 \mathrm{~Hz}, \mathrm{H}-3\right), 4.61$ (d, $\left.1 \mathrm{H}, J_{1,2}=10.0 \mathrm{~Hz}, \mathrm{H}-1\right)$, 4.19-4.01 (complex mixture of signals, 7H), 3.64-3.57 (m, 4H), 3.48 (bs, 3H), 3.36-3.26 (mixture of singlets, $4 \mathrm{H}), 3.16(\mathrm{~m}, 1 \mathrm{H}), 3.01(\mathrm{~m}, 1 \mathrm{H}), 2.17-1.98$ (mixture of singlets, $12 \mathrm{H}, \mathrm{Ac})$.

${ }^{13} \mathrm{C} \mathrm{NMR}\left(\mathrm{CDCl}_{3}, 75 \mathrm{MHz}\right) \delta 170.5,170.3,170.1,169.7,164.1,163.8,83.8,74.8,71.7,70.4,67.3$, $66.7,61.5,58.9,51.4,51.0,46.8,46.3,27.0,20.8,20.7,20.6,20.5$.

MS (ESI): $m / z(\%)=(100)[\mathrm{M}+\mathrm{H}]^{+}$. HRMS (ESI): calcd. for $\mathrm{C}_{23} \mathrm{H}_{36} \mathrm{~N}_{2} \mathrm{O}_{13} \mathrm{~S}[\mathrm{M}+\mathrm{H}]^{+} 581.2016$;

found 581.2013. Isolated yield obtained by the use of TGT resin $0.16 \mathrm{mmol} / \mathrm{g}$ (entry $f$ fig. S2): $95 \%$ as a white solid.

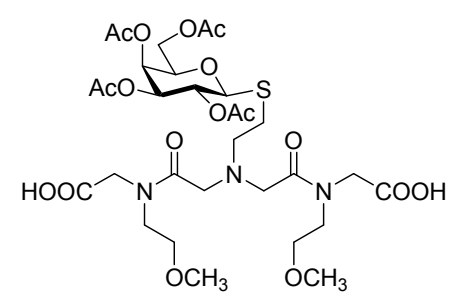

(2): ${ }^{1} \mathrm{H} \mathrm{NMR}\left(\mathrm{CDCl}_{3}, 400 \mathrm{MHz}\right.$, mixture of rotamers) $\delta 5.43$ (bs, $\left.1 \mathrm{H}, \mathrm{H}-4\right), 5.17-5.08$ (m, 2H, H-2, H-3), 4.70-4.65 (m, 1H, H-1), 4.59-4.30 (complex mixture of signals, 4H), 4.16-4.04 (complex mixture of signals, $7 \mathrm{H}), 3.59(\mathrm{~m}, 4 \mathrm{H}), 3.49(\mathrm{bs}, 6 \mathrm{H}), 3.31-3.26$ (mixture of singlets, $6 \mathrm{H}), 3.08(\mathrm{~m}$, 1H), $2.96(\mathrm{~m}, 1 \mathrm{H}), 2.16-1.97$ (mixture of singlets, $12 \mathrm{H}, \mathrm{Ac}$ ).

${ }^{13} \mathrm{C} \mathrm{NMR}\left(\mathrm{CDCl}_{3}, 75 \mathrm{MHz}\right.$, mixture of rotamers) $\delta 170.8,170.7,170.28,170.23,170.18,170.1$,

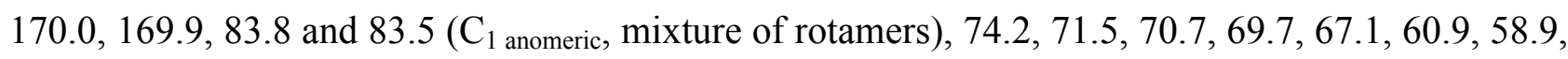
$58.4,56.1,56.0,55.6,50.1,48.5,48.0,47.8,24.8,20.6$.

MS (ESI): $m / z(\%)=(100)[\mathrm{M}+\mathrm{H}]^{+}$. HRMS (ESI): calcd. for $\mathrm{C}_{30} \mathrm{H}_{47} \mathrm{~N}_{3} \mathrm{O}_{17} \mathrm{~S}[\mathrm{M}+\mathrm{H}]^{+} 754.2704$;

found 754.2707 . Isolated yield obtained by the use of 2-Chlorotrityl resin $1.6 \mathrm{mmol} / \mathrm{g}$ (entry $a$ fig. S1): $72 \%$ as a white solid. 


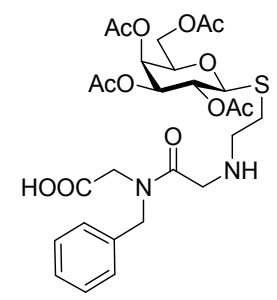

(3): ${ }^{1} \mathrm{H} \mathrm{NMR}\left(\mathrm{CDCl}_{3}, 400 \mathrm{MHz}\right.$, mixture of rotamers) $\delta 7.39-7.19(\mathrm{~m}, 5 \mathrm{H}, \mathrm{Ar}), 5.45(1 \mathrm{H}, \mathrm{H}-4), 5.27-$ $5.23(1 \mathrm{H}, \mathrm{H}-2), 5.08-5.06(1 \mathrm{H}, \mathrm{H}-3), 4.70-4.50(3 \mathrm{H}), 4.21-4.76$ (complex mixture of signals, $7 \mathrm{H}$ ), 3.35-3.00 (m, 4H), 2.17-1.98 (mixture of singlets, 12H, Ac).

${ }^{13} \mathrm{C} \mathrm{NMR}\left(\mathrm{CDCl}_{3}, 75 \mathrm{MHz}\right) \delta 170.5,170.3,170.1,169.8,163.7,163.6,129.1(\times 2), 128.6(\times 2)$, 128.4, 127.0, 83.8, 74.8, 71.7, 67.3, 66.7, 61.5, 51.0, 49.6, 49.0, 46.8, 26.9, 20.7, 20.6, 20.5, 20.4. MS (ESI): $m / z(\%)=(100)[\mathrm{M}+\mathrm{H}]^{+}$. HRMS (ESI): calcd. for $\mathrm{C}_{27} \mathrm{H}_{36} \mathrm{~N}_{2} \mathrm{O}_{12} \mathrm{~S}[\mathrm{M}+\mathrm{H}]^{+} 613.2067$; found 613.2155 . Isolated yield obtained by the use of 2-Chlorotrityl resin $0.1 \mathrm{mmol} / \mathrm{g}$ (entry $d$ fig. S3): $95 \%$ as a white solid.

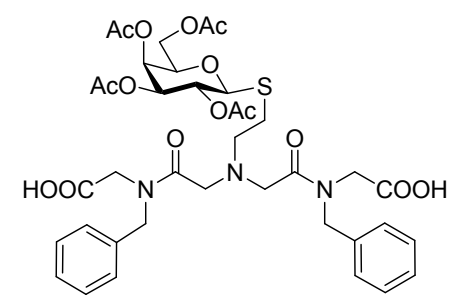

(4): ${ }^{1} \mathrm{H} \mathrm{NMR}\left(\mathrm{CDCl}_{3}, 400 \mathrm{MHz}\right.$, mixture of rotamers) $\delta 7.33-7.18(\mathrm{~m}, 10 \mathrm{H}, \mathrm{Ar}), 5.41(1 \mathrm{H}, \mathrm{H}-4)$, 5.23-5.14 (1H, H-2), 5.07 (1H, H-3), 4.63-4.38 (9H), 4.15-3.96 (complex mixture of signals, 7H), $3.64(\mathrm{~m}, 2 \mathrm{H}), 3.18-3.08(\mathrm{~m}, 1 \mathrm{H}), 3.01-2.92(\mathrm{~m}, 1 \mathrm{H}), 2.10-1.93$ (mixture of singlets, $12 \mathrm{H}, \mathrm{Ac}$ ).

${ }^{13} \mathrm{C} \mathrm{NMR}\left(\mathrm{CDCl}_{3}, 75 \mathrm{MHz}\right) \delta 170.8,170.7,170.3,170.2,170.0,169.9,165.9,165.6,135.0,134.9$, $134.3,134.0,129.2,128.9(\times 2), 128.5,128.3,128.2,128.1,127.3,83.7$ and $83.1\left(\mathrm{C}_{1}\right.$ anomeric, mixture of rotamers), 74.6, 74.4, 71.5, 67.1, 66.9, 61.0, 56.4, 51.0, 47.9, 25.0, 24.6, 20.7, 20.6, 20.5, 20.4 .

MS (ESI): $m / z(\%)=(100)[\mathrm{M}+\mathrm{H}]^{+}$. HRMS (ESI): calcd. for $\mathrm{C}_{38} \mathrm{H}_{47} \mathrm{~N}_{3} \mathrm{O}_{15} \mathrm{~S}[\mathrm{M}+\mathrm{H}]^{+} 818.2806$; found 818.2925 . Isolated yield obtained by the use of 2-Chlorotrityl resin $1.6 \mathrm{mmol} / \mathrm{g}$ (entry $a$ fig. S3): $25 \%$ as a white solid.

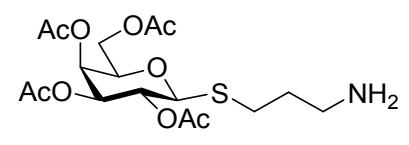

Synthesis of 3-Aminopropyl 2,3,4,6-penta- $O$-acetyl-1-thio- $\beta$-D-galactopyranoside: 
$\beta$-D-galactose pentaacetate $(6.00 \mathrm{mmol})$ in DCM $(20 \mathrm{~mL})$ was treated with iodine $(2.13 \mathrm{~g}, 8.40 \mathrm{mmol})$ and $\mathrm{Et}_{3} \mathrm{SiH}(1.34 \mathrm{~mL}, 8.40 \mathrm{mmol}) .{ }^{3}$ The mixture was refluxed for few minutes, then diluted with $\operatorname{DCM}(100 \mathrm{~mL})$ and washed with $1: 1 \mathrm{v} / \mathrm{v} 1 \mathrm{M} \mathrm{NaHCO} / 10 \% \mathrm{Na}_{2} \mathrm{~S}_{2} \mathrm{O}_{3}(100 \mathrm{~mL})$ and water $(100 \mathrm{~mL})$. The organic layer was collected, dried over anhydrous $\mathrm{Na}_{2} \mathrm{SO}_{4}$, filtered and concentrated. The residue was mixed with thiourea $(685 \mathrm{mg}, 900 \mathrm{mmol})$, suspended in $\mathrm{ACN}(10 \mathrm{~mL})$ and then heated to $60^{\circ} \mathrm{C} .{ }^{4}$ After $20 \mathrm{~min}$ the reaction mixture was cooled to room temperature, then TEA (3.34 $\mathrm{mL}, 24.0 \mathrm{mmol})$ and 2-bromopropylamine hydrobromide (1.96 g, $8.98 \mathrm{mmol})$ were added. The mixture was stirred for an additional 1 hour and then diluted with $\mathrm{DCM}(100 \mathrm{~mL})$ and washed with sat. $\mathrm{NaHCO}_{3}$. The organic layer was collected, dried over anhydrous $\mathrm{Na}_{2} \mathrm{SO}_{4}$, filtered, concentrated and subjected to column chromatography.

Column chromatography ( $4 \%$ to $10 \% \mathrm{MeOH}$ in DCM with $0.1 \%$ of $\mathrm{NH}_{4} \mathrm{OH}$ ); colourless foam; isolated yield: $55 \%$ from compound $\beta$-D-galactose pentacetate.

$[\alpha]_{\mathrm{D}}\left(c, \mathrm{CHCl}_{3}\right) .{ }^{1} \mathrm{H} \mathrm{NMR}\left(\mathrm{CDCl}_{3}, 400 \mathrm{MHz}\right) \delta 5.42\left(\mathrm{~d}, 1 \mathrm{H}, J_{4,3}=3.3 \mathrm{~Hz}, \mathrm{H}-4\right), 5.21\left(\mathrm{t}, 1 \mathrm{H}, J_{2,3}=\right.$ $\left.J_{2,1}=10.0 \mathrm{~Hz}, \mathrm{H}-2\right), 5.05\left(\mathrm{dd}, 1 \mathrm{H}, J_{3,2}=10.0, J_{3,4}=3.3 \mathrm{~Hz}, \mathrm{H}-3\right), 4.56\left(\mathrm{~d}, 1 \mathrm{H}, J_{1,2}=10.0 \mathrm{~Hz}, \mathrm{H}-1\right), 4.26$ $\left(\mathrm{dd}, 1 \mathrm{H}, J_{\mathrm{gem}}=11.3 \mathrm{~Hz}, J_{6,5}=6.4 \mathrm{~Hz}, \mathrm{H}-6 \mathrm{a}\right), 4.08\left(\mathrm{dd}, 1 \mathrm{H}, J_{\mathrm{gem}}=11.3 \mathrm{~Hz}, J_{6,5}=6.4 \mathrm{~Hz}, \mathrm{H}-6 \mathrm{~b}\right), 4.01$ (t, $\left.1 \mathrm{H}, J_{5,6}=6.4 \mathrm{~Hz}, \mathrm{H}-5\right), 3.07\left(\mathrm{~m}, 2 \mathrm{H},-\mathrm{CH}_{2}-\right), 2.93-2.86(\mathrm{~m},-\mathrm{CHH}-, 1 \mathrm{H}), 2.80-2.73(\mathrm{~m},-\mathrm{CHH}-, 1 \mathrm{H})$, $2.16\left(\mathrm{~s}, 3 \mathrm{H},-\mathrm{COCH}_{3}\right), 2.06\left(\mathrm{~s}, 6 \mathrm{H}, 2 \times-\mathrm{COCH}_{3}\right), 2.04-1.98\left(\mathrm{~m}, 2 \mathrm{H},-\mathrm{CH}_{2}-\right), 1.97\left(\mathrm{~s}, 3 \mathrm{H},-\mathrm{COCH}_{3}\right)$; ${ }^{13} \mathrm{C} \mathrm{NMR}\left(\mathrm{CDCl}_{3}, 75 \mathrm{MHz}\right) \delta 170.8,170.2,170.0,167.8,83.7,74.6,71.7,67.3,67.0,61.6,39.5$, 29.2, 27.1, 20.9, 20.8, 20.7, 20.5 .

MS (ESI): $m / z(\%)=(100)[\mathrm{M}+\mathrm{H}]^{+}$. HRMS (ESI): calcd. for $\mathrm{C}_{17} \mathrm{H}_{28} \mathrm{NO}_{9} \mathrm{~S}[\mathrm{M}+\mathrm{H}]^{+} 422.1485$; found 422.1490 .

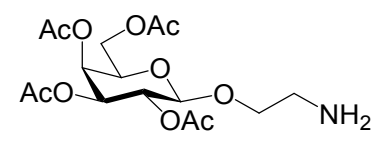

\section{Synthesis of 2-Aminoethyl 2,3,4,6-penta- $O$-acetyl-1- $O$ - $\beta$-D-galactopyranoside:}

$\beta$-D-galactose pentaacetate $(1.28 \mathrm{mmol})$ in DCM dry $(4 \mathrm{~mL})$ was treated with $\mathrm{BF}_{3} \cdot \mathrm{OEt}_{2}(610 \mu 1,5.12$ mmol) and 2-bromoethanol $(110 \mu \mathrm{l}, 1.54 \mathrm{mmol})$ at $0^{\circ} \mathrm{C}$. The mixture was stirred for $3 \mathrm{~h}$ at $\mathrm{rt}$, then treated with $\mathrm{K}_{2} \mathrm{CO}_{3}$ anhydrous (1.92 mmol, $250 \mathrm{mg}$ ) for $30 \mathrm{~min}$ and then diluted with DCM (100 mL) and washed with water $(100 \mathrm{~mL})$. The organic layer was collected, dried over anhydrous $\mathrm{Na}_{2} \mathrm{SO}_{4}$, filtered, concentrated and subjected to column chromatography (petrolium ether/ethyl acetate 70:30, $75 \%$ yield) to obtain 2-bromo 2,3,4,6-penta- $O$-acetyl-1- $O$ - $\beta$-D-galactopyranoside ${ }^{5}$. The glycosyl

\footnotetext{
${ }^{3}$ Adinolfi, M.; Iadonisi, A.; Ravidà, A.; Schiattarella, M. Tetrahedron Lett. 2003, 44, 7863-7866.

${ }^{4}$ Valerio, S.; Iadonisi, A.; Adinolfi, M.; Ravidà, A. J. Org. Chem 2007, 72, 6097-6106.

${ }^{5}$ Yarlagadda, V. et al The Journal of Antibiotics 2014, 1-11.
} 
bromide $(1 \mathrm{mmol})$ was dissolved in methanol $(5 \mathrm{ml})$ and treated with $\mathrm{NaN}_{3}(1.9 \mathrm{mmol}, 124 \mathrm{mg})$ at $70^{\circ} \mathrm{C}$ overnight, then diluted with DCM $(100 \mathrm{~mL})$ and washed with brine $(100 \mathrm{~mL})$. The organic layer was collected, dried over anhydrous $\mathrm{Na}_{2} \mathrm{SO}_{4}$, filtered, concentrated and subjected to column chromatography (petrolium ether/ethyl acetate 70:30, 60\% yield) to obtain 2-azide 2,3,4,6-penta- $O$ acetyl-1-O- $\beta$-D-galactopyranoside ${ }^{5}$. The azide $(1 \mathrm{mmol})$ was dissolved in methanol $(5 \mathrm{ml})$ and treated with $\mathrm{Pd} / \mathrm{C} 10 \mathrm{wt} \%(78 \mathrm{mg})$ in hydrogen atmosphere for $2 \mathrm{~h}$ at $\mathrm{rt}$. The mixture was filtered on silica gel, dried and subjected to column chromatography (DCM/MeOH 90:10) to obtain 2-amino 2,3,4,6penta- $O$-acetyl-1-O- $\beta$-D-galactopyranoside (isolated yield: $40 \%$ ) as a colourless foam.

MS (ESI): $m / z(\%)=(100)[\mathrm{M}+\mathrm{H}]^{+}$. HRMS (ESI): calcd. for $\mathrm{C}_{16} \mathrm{H}_{26} \mathrm{NO}_{10}[\mathrm{M}+\mathrm{H}]^{+}$392.1557; found 392.1563 . 


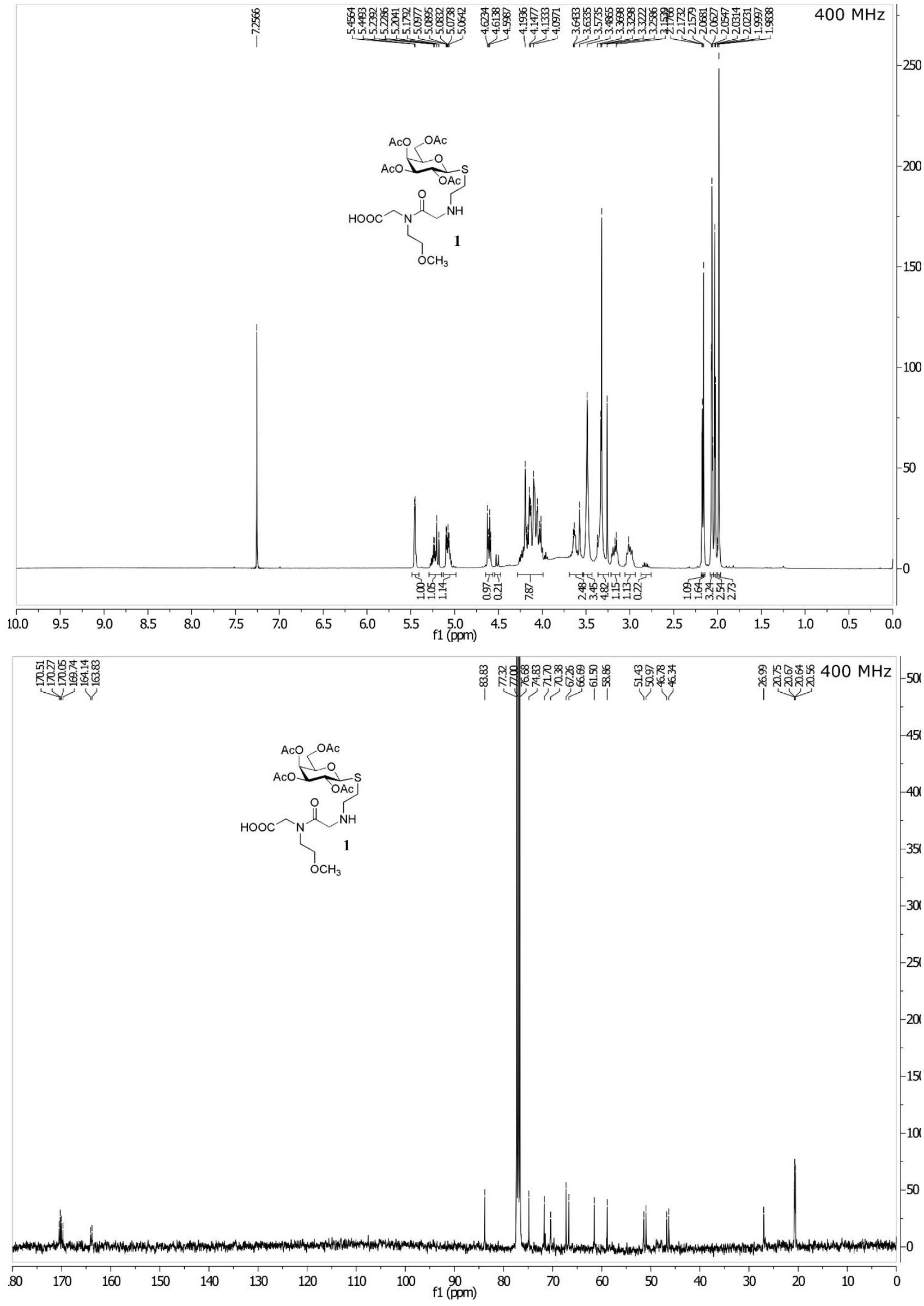




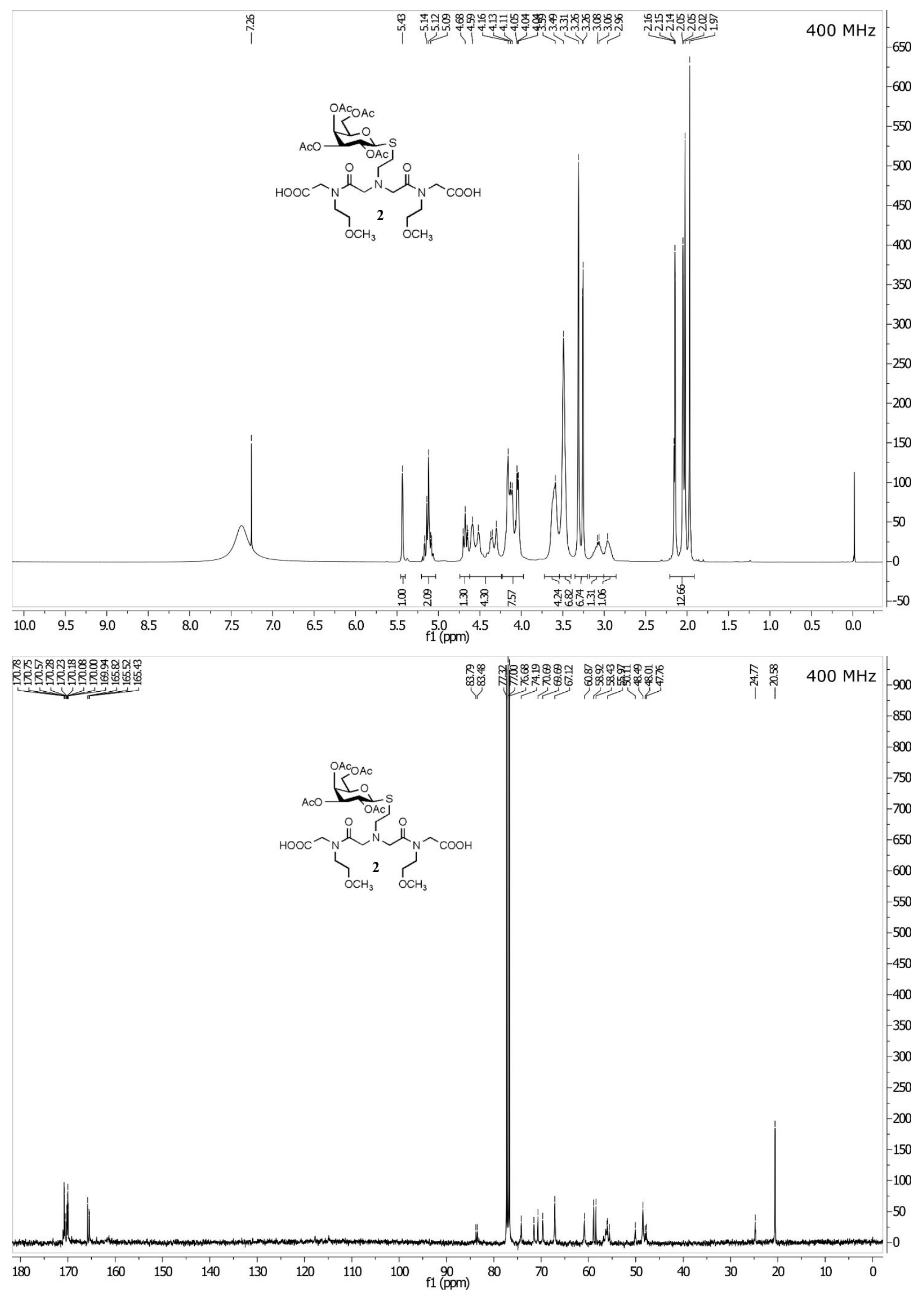




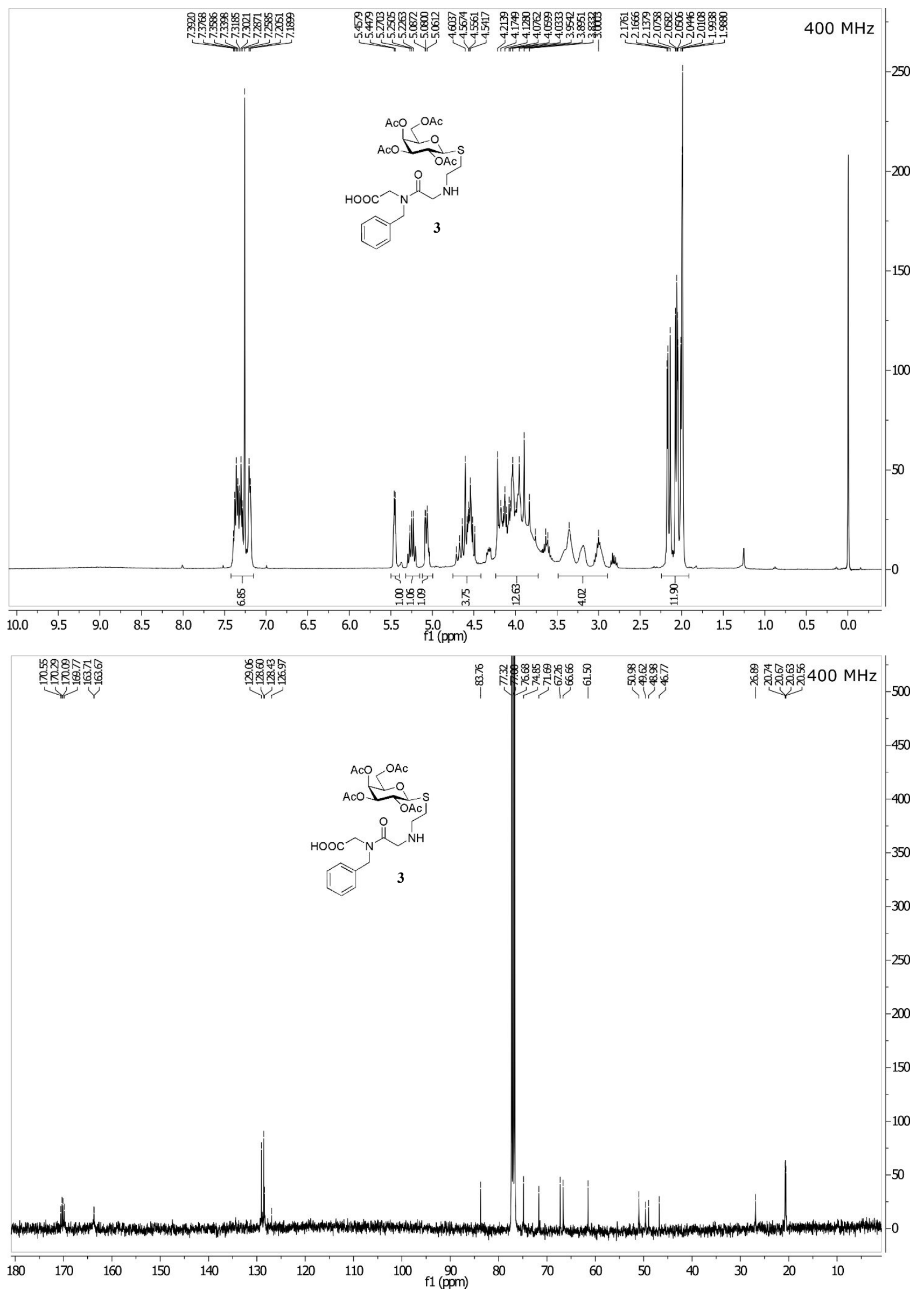




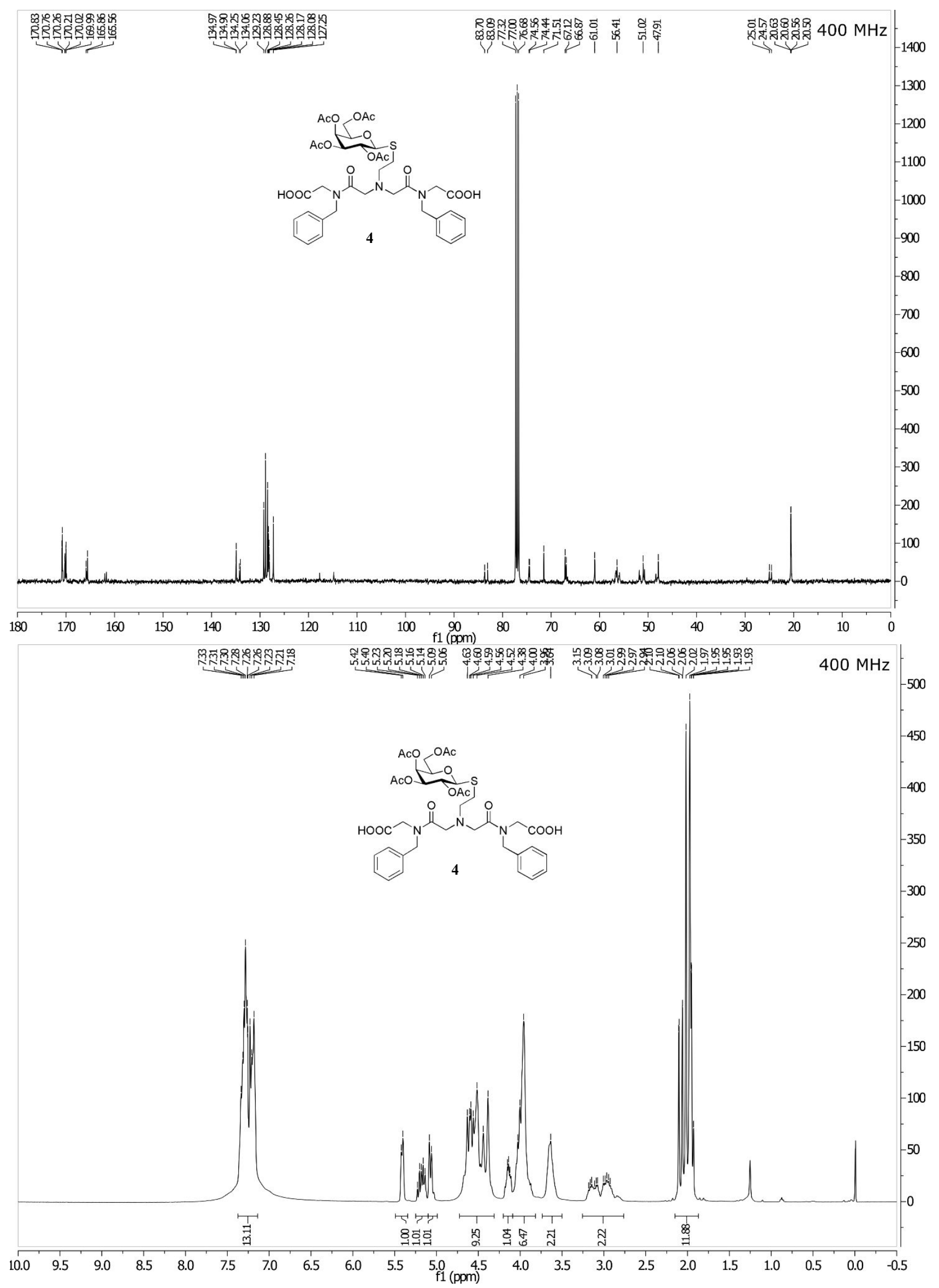




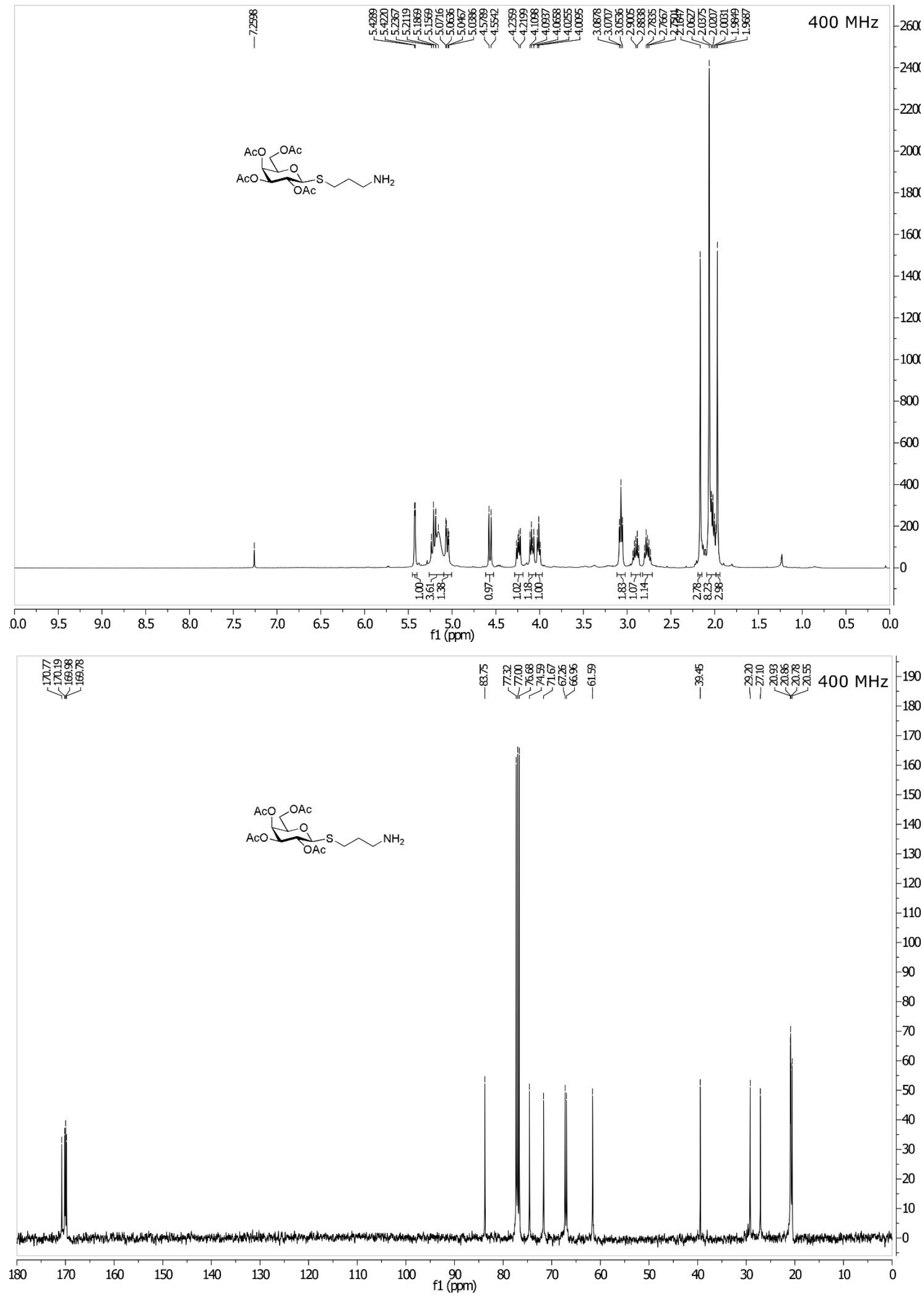




\section{LC-MS Analysis and Integration Peak Lists}

Fig S1: Nme-N(ethyl 2,3,4,6-penta-O-acetyl-1-S-beta-D-galactosyl)glycine (Ngalte) dipeptoid crudes from the submonomer synthesis by the use of different loading of 2-Chlorotrityl resin (i.e.: $1.6 \mathrm{mmol} / \mathrm{g}$ entry a; $1.0 \mathrm{mmol} / \mathrm{g}$ entry $\mathrm{b} ; 0.6 \mathrm{mmol} / \mathrm{g}$ entry c; $0.1 \mathrm{mmol} / \mathrm{g}$ entry d)

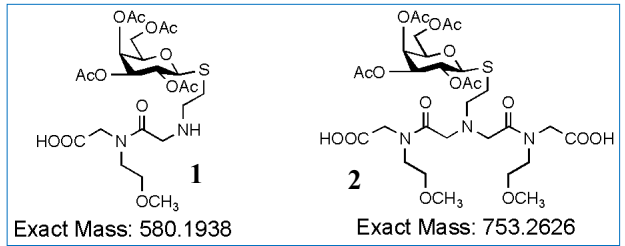

Entry a

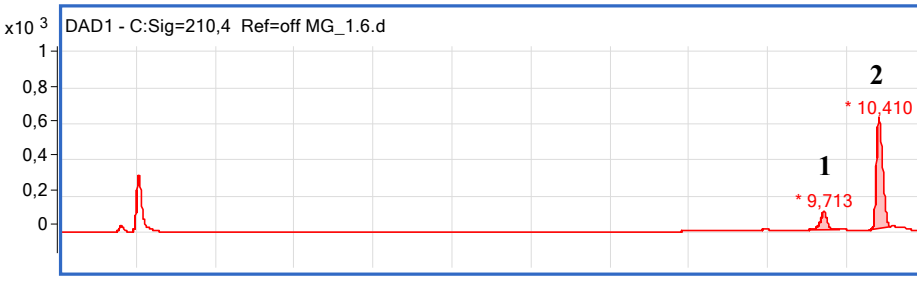

Entry b

Entry c

Entry d
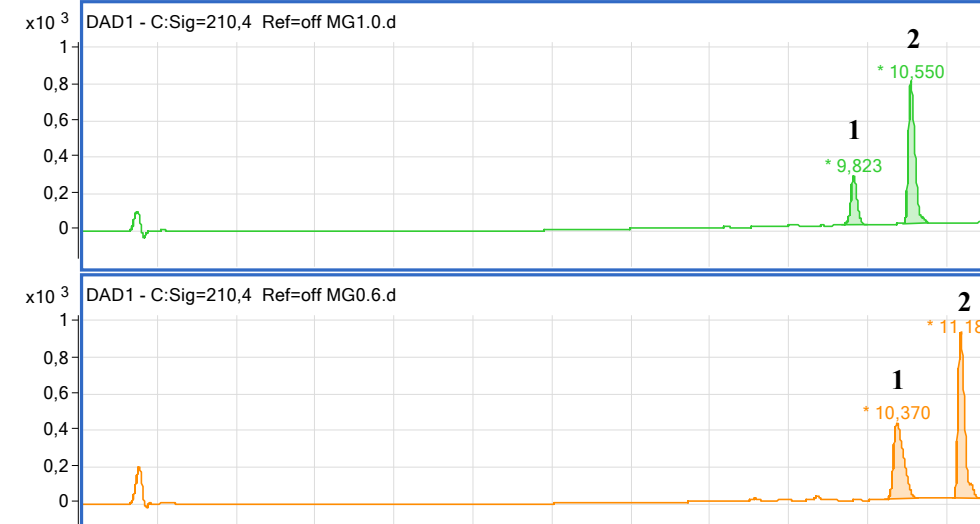

x10 $1{ }^{1}$ DAD1 - C:Sig=210,4 Ref=off MG0.1.d

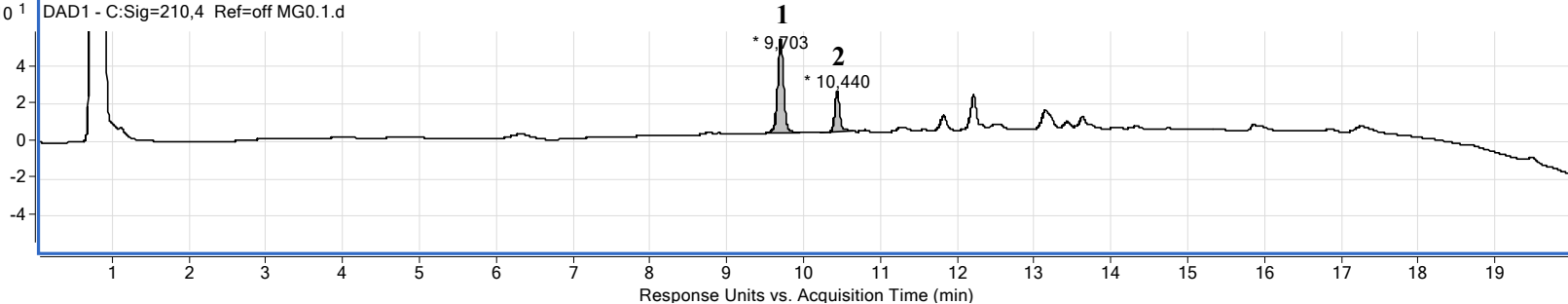

\begin{tabular}{|c|c|c|c|c|c|c|c|}
\hline \multicolumn{8}{|c|}{ Entry a } \\
\hline Peak & MW & RT & Area & Height & Width & $\begin{array}{l}\text { Integratio } \\
\text { n ratio } \\
\text { (\%) }\end{array}$ & $\begin{array}{l}\text { Real } \\
\text { ratio } \\
(\%)\end{array}$ \\
\hline 1 & 581.2108 & 9.713 & 600.9 & 104.2 & 0.29 & \multirow[t]{2}{*}{$14 / 86$} & \multirow[t]{2}{*}{$25 / 75$} \\
\hline 2 & 754.2691 & 10.410 & 3573.5 & 621.6 & 0.29 & & \\
\hline
\end{tabular}




\begin{tabular}{|c|c|c|c|c|c|c|c|}
\hline Peak & MW & RT & Area & Height & Width & $\begin{array}{l}\text { Integratio } \\
\text { n ratio } \\
(\%)\end{array}$ & $\begin{array}{l}\text { Real } \\
\text { ratio } \\
(\%)\end{array}$ \\
\hline 1 & 581.2108 & 9.823 & 1458.9 & 266.4 & 0.36 & \multirow[t]{2}{*}{$25 / 75$} & \multirow[t]{2}{*}{$40 / 60$} \\
\hline 2 & 754.2691 & 10.550 & 4511.5 & 775.1 & 0.44 & & \\
\hline \multicolumn{8}{|c|}{ Entry c } \\
\hline Peak & MW & RT & Area & Height & Width & $\begin{array}{l}\text { Integration } \\
\text { ratio }(\%)\end{array}$ & $\begin{array}{l}\text { Real } \\
\text { ratio } \\
(\%)\end{array}$ \\
\hline 1 & 581.2108 & 10.370 & 3653.8 & 414.0 & 0.41 & \multirow[t]{2}{*}{$42 / 58$} & \multirow[t]{2}{*}{$60 / 40$} \\
\hline 2 & 754.2691 & 11.180 & 5114.0 & 897.7 & 0.41 & & \\
\hline \multicolumn{8}{|c|}{ Entryd } \\
\hline Peak & MW & RT & Area & Height & Width & $\begin{array}{l}\text { Integration } \\
\text { ratio }(\%)\end{array}$ & $\begin{array}{l}\text { Real } \\
\text { ratio } \\
(\%)\end{array}$ \\
\hline 1 & 581.2108 & 9.703 & 276.7 & 50.4 & 0.46 & \multirow[t]{2}{*}{$72 / 28$} & \multirow[t]{2}{*}{$84 / 16$} \\
\hline 2 & 754.2691 & 10.440 & 106.4 & 21.8 & 0.43 & & \\
\hline
\end{tabular}

Fig S2: Nme-N(ethyl 2,3,4,6-penta-O-acetyl-1-S-beta-D-galactosyl)glycine (Ngalte) dipeptoid crudes from the submonomer synthesis on TGR (entry e) and TGT (entryf) resins

Entrye

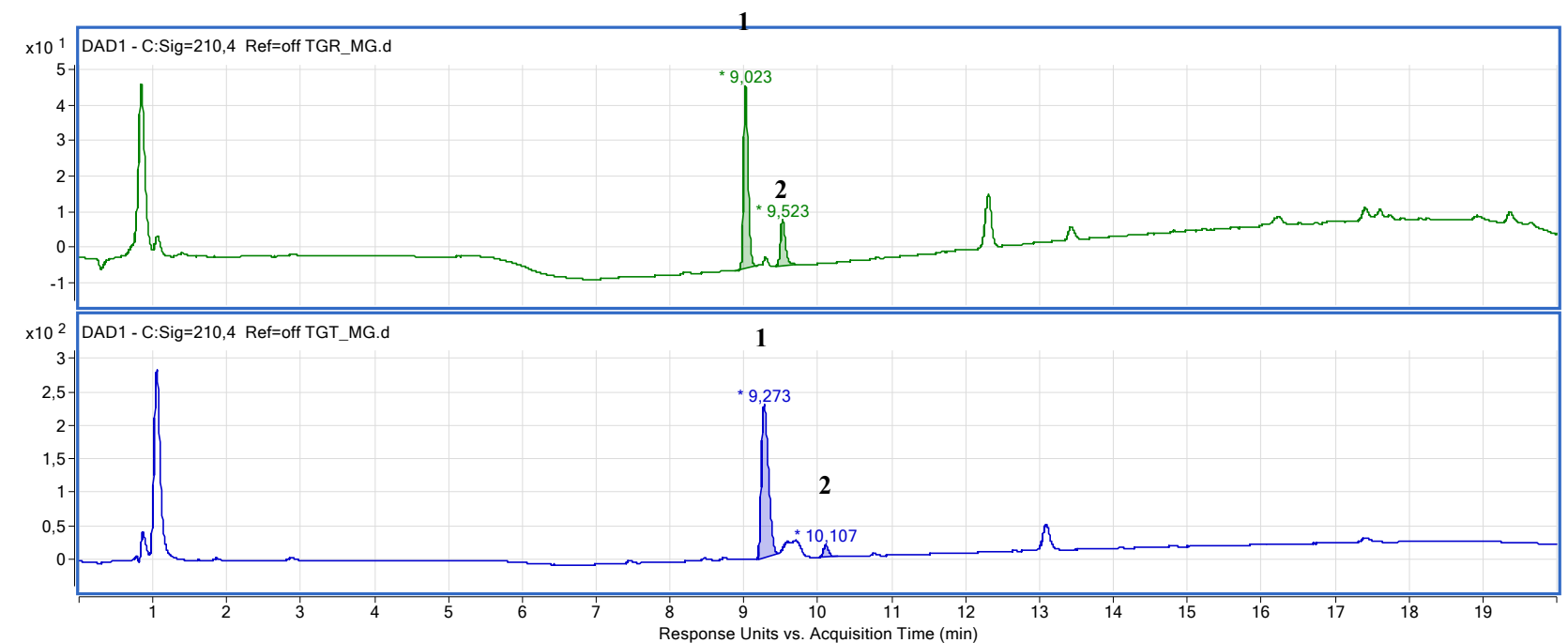

\begin{tabular}{|c|c|c|c|c|c|c|c|}
\hline Entrye & & & & & & & \\
\hline Peak & MW & RT & Area & Height & Width & $\begin{array}{l}\text { Integration } \\
\text { ratio (\%) }\end{array}$ & $\begin{array}{l}\text { Real ratio } \\
(\%)\end{array}$ \\
\hline 1 & 580.2260 & 9.023 & 222.8 & 51.3 & 0.32 & \multirow[t]{2}{*}{$79 / 21$} & \multirow[t]{2}{*}{$88 / 12$} \\
\hline 2 & 752.3135 & 9.523 & 60.1 & 12.8 & 0.28 & & \\
\hline \multicolumn{8}{|l|}{ Entryf } \\
\hline Peak & MW & RT & Area & Height & Width & $\begin{array}{l}\text { Integration } \\
\text { ratio }(\%)\end{array}$ & $\begin{array}{l}\text { Real ratio } \\
(\%)\end{array}$ \\
\hline 1 & 581.2042 & 9.273 & 1511.3 & 228.2 & 0.28 & \multirow[t]{2}{*}{$95 / 5$} & \multirow{2}{*}{$97 / 3$} \\
\hline 2 & 754.2740 & 10.107 & 79.1 & 17.9 & 0.26 & & \\
\hline
\end{tabular}


Fig S3: Npm-N(ethyl 2,3,4,6-penta-O-acetyl-1-S-beta-D-galactosyl)glycine (Ngalte) dipeptoid crudes from the submonomer synthesis by the use of different loading of 2-Chlorotrityl resin (i.e.: $1.6 \mathrm{mmol} / \mathrm{g}$ entry a; $1.0 \mathrm{mmol} / \mathrm{g}$ entry b; $0.6 \mathrm{mmol} / \mathrm{g}$ entry c; 0.1 $\mathrm{mmol} / \mathrm{g}$ entry d)

Entry a
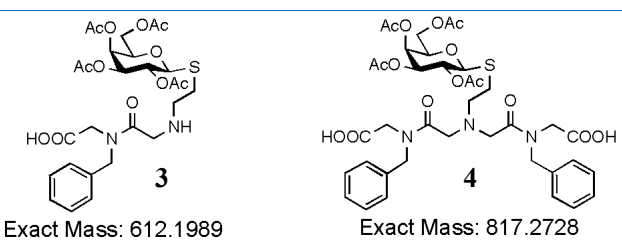

Entry $b$

Entry c

Entryd
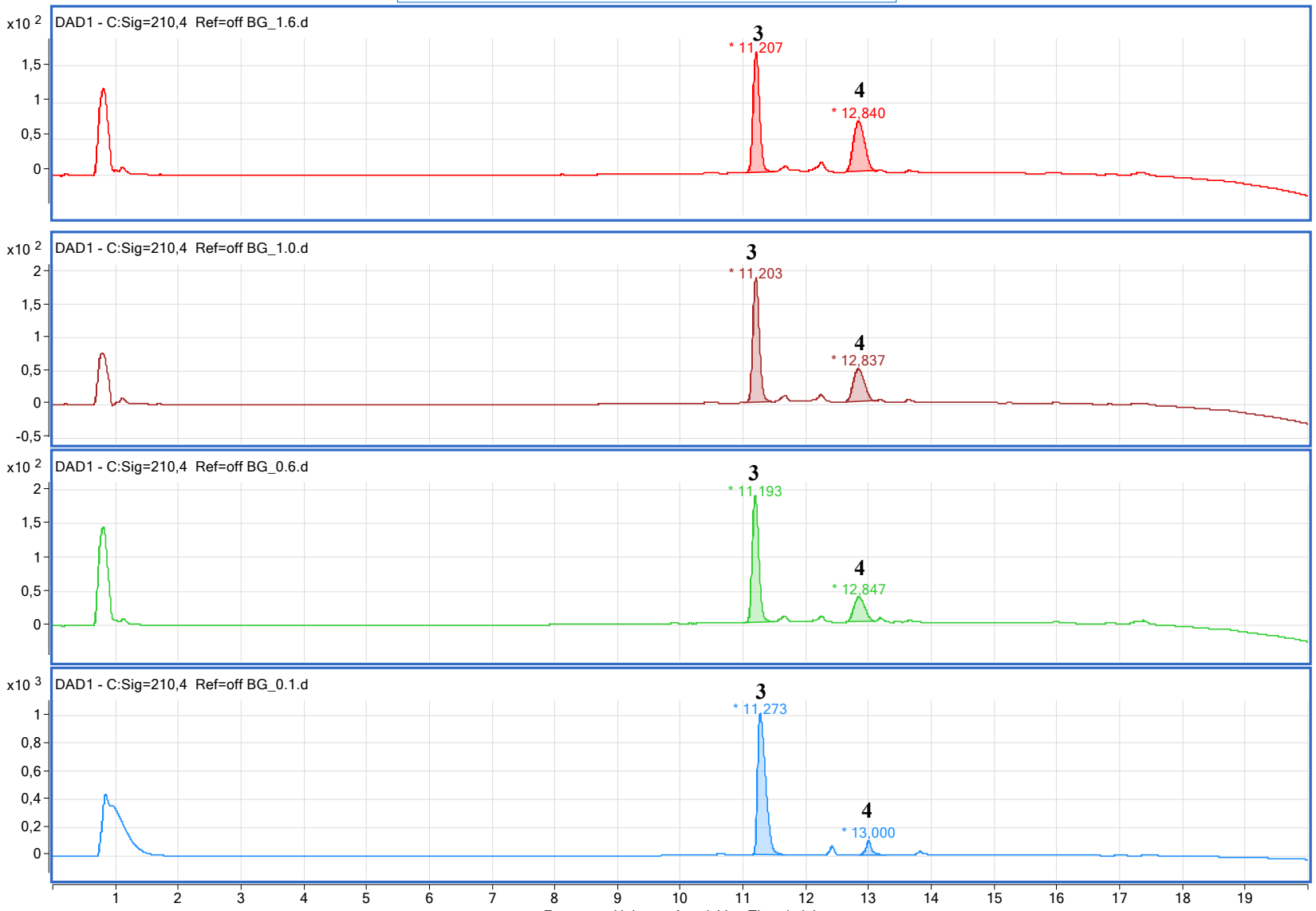

\begin{tabular}{|c|c|c|c|c|c|c|c|}
\hline \multicolumn{8}{|c|}{ Entry a } \\
\hline Peak & MW & RT & Area & Height & Width & $\begin{array}{l}\text { Integratio } \\
\text { n ratio } \\
\text { (\%) }\end{array}$ & $\begin{array}{l}\text { Real ratio } \\
(\%)\end{array}$ \\
\hline 3 & 613.0635 & 11.207 & 1184.1 & 164.1 & 0.423 & \multirow[t]{2}{*}{$58 / 42$} & \multirow[t]{2}{*}{$73 / 27$} \\
\hline 4 & 818.1089 & 12.840 & 877.6 & 69.8 & 0.533 & & \\
\hline \multicolumn{8}{|c|}{ Entry b } \\
\hline Peak & MW & RT & Area & Height & Width & $\begin{array}{l}\text { Integratio } \\
\text { n ratio } \\
(\%)\end{array}$ & $\begin{array}{l}\text { Real ratio } \\
(\%)\end{array}$ \\
\hline 3 & 613.0635 & 11.203 & 1344.1 & 184.8 & 0.45 & \multirow[t]{2}{*}{$70 / 30$} & \multirow[t]{2}{*}{$83 / 17$} \\
\hline 4 & 818.1089 & 12.837 & 593.1 & 49.1 & 0.52 & & \\
\hline \multicolumn{8}{|c|}{ Entry c } \\
\hline Peak & MW & RT & Area & Height & Width & $\begin{array}{l}\text { Integratio } \\
\text { n ratio } \\
(\%)\end{array}$ & $\begin{array}{l}\text { Real ratio } \\
(\%)\end{array}$ \\
\hline 3 & 613.0635 & 11.193 & 1343.5 & 184.3 & 0.517 & $75 / 25$ & $85 / 15$ \\
\hline
\end{tabular}




\begin{tabular}{|c|c|c|c|c|c|c|c|}
\hline 4 & 818.1089 & 12.847 & 429.2 & 35.7 & 0.453 & & \\
\hline \multicolumn{8}{|c|}{ Entryd } \\
\hline Peak & MW & RT & Area & Height & Width & $\begin{array}{l}\text { Integratio } \\
\text { n ratio } \\
\text { (\%) }\end{array}$ & $\begin{array}{l}\text { Real ratio } \\
(\%)\end{array}$ \\
\hline 3 & 613.0635 & 11.273 & 8863.6 & 991.1 & 0.50 & \multirow[t]{2}{*}{$93 / 7$} & \multirow[t]{2}{*}{$96 / 4$} \\
\hline 4 & 818.1089 & 13.000 & 666.5 & 104.0 & 0.44 & & \\
\hline
\end{tabular}

Fig. S4: Full submonomer synthesis of hexamer 5 on Novasyn TGT resin (Loading $0.16 \mathrm{mmol} / \mathrm{g}$ )

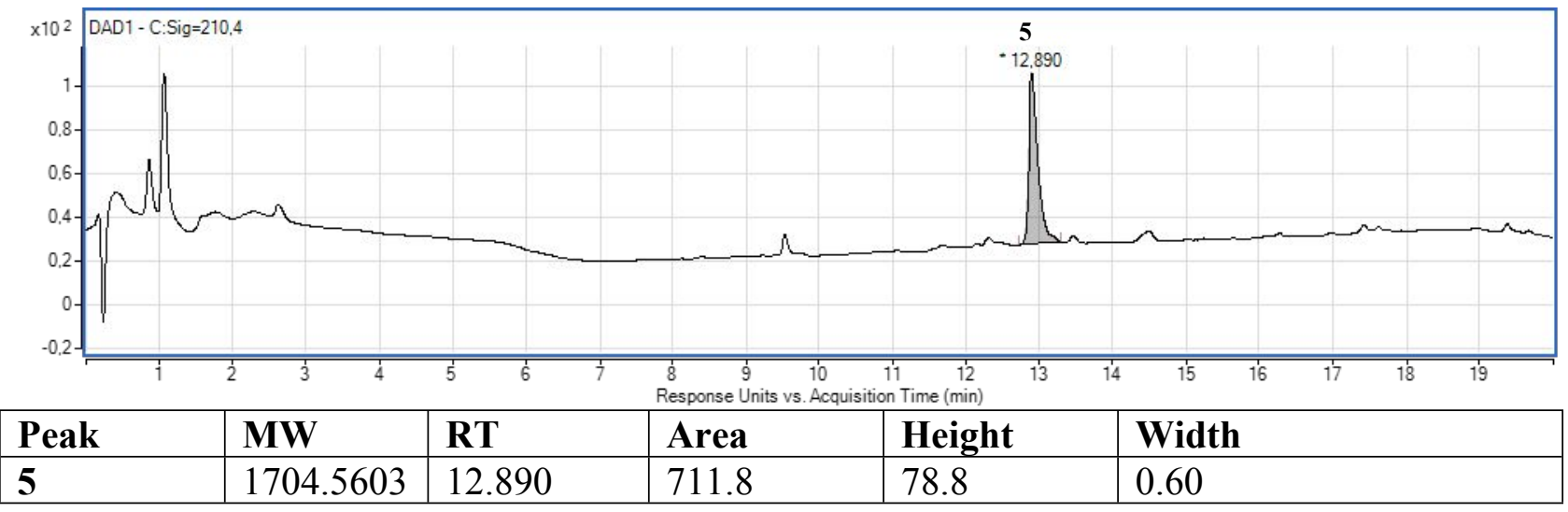

Fig S1: Distribution of byproducts from the submonomer synthesis of the pentapeptoid 7 on 2-Cholorotrityl resin (Loading 1.6 $\mathrm{mmol} / \mathrm{g}$ )
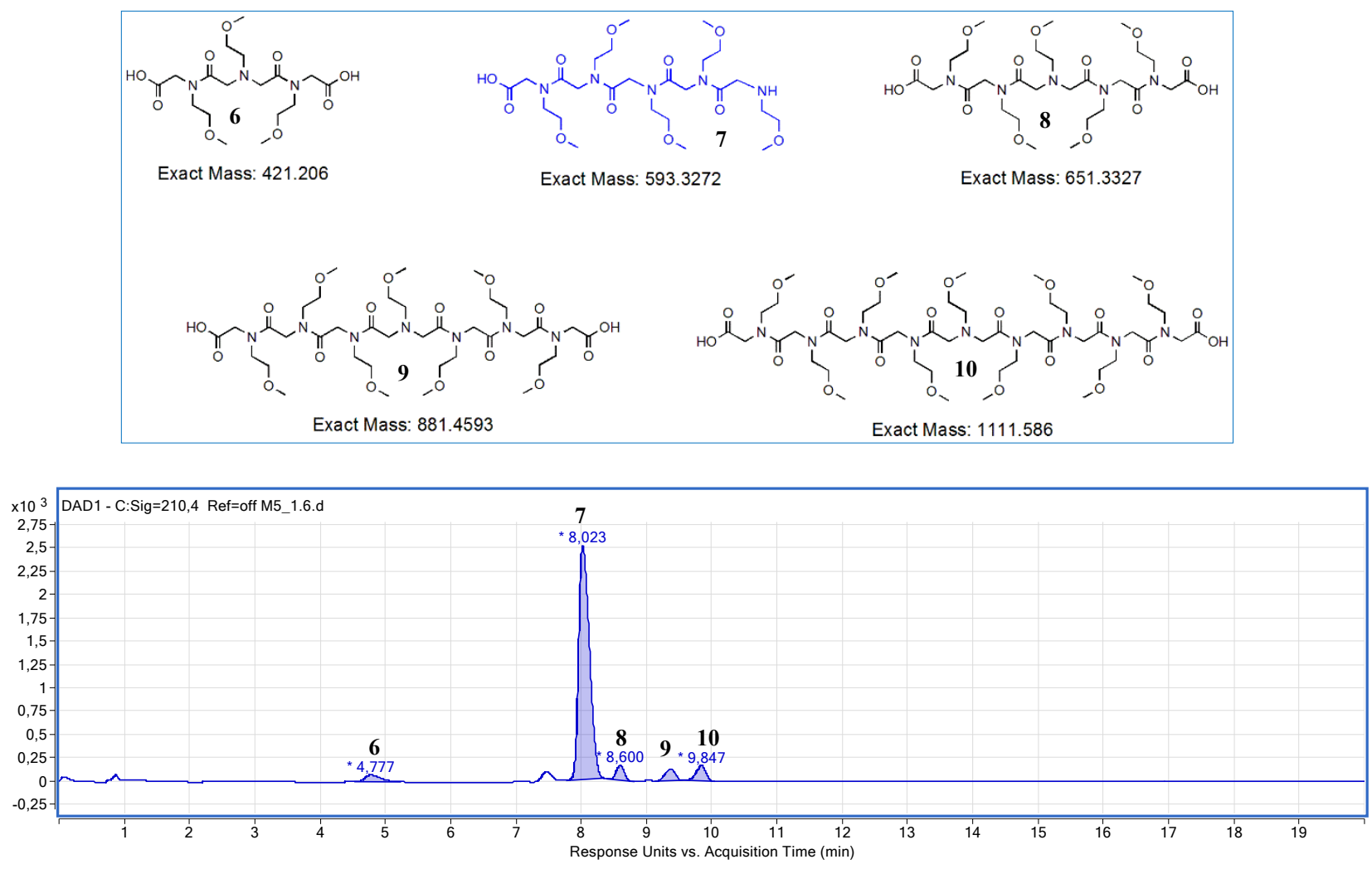


\begin{tabular}{|c|c|c|c|c|c|c|c|}
\hline Peak & MW & RT & Area & Height & Width & $\begin{array}{l}\text { Integration } \\
\text { ratio }(\%)\end{array}$ & $\begin{array}{l}\text { Real } \\
\text { ratio } \\
(\%)\end{array}$ \\
\hline 6 & 422.2171 & 4.777 & 1140.6 & 72.3 & 0.62 & \multirow[t]{5}{*}{$94 / 6$} & \multirow[t]{5}{*}{$97 / 3$} \\
\hline 7 & 594.3425 & 8.023 & 28954.5 & 2508.9 & 0.68 & & \\
\hline 8 & 652.3465 & 8.6 & 1342.7 & 159.7 & 0.34 & & \\
\hline 9 & 882.4762 & 9.373 & 1208.4 & 120.3 & 0.51 & & \\
\hline 10 & 1112.6052 & 9.847 & 1713.7 & 163.4 & 0.51 & & \\
\hline
\end{tabular}

Fig S6: Distribution of byproducts from the submonomer synthesis of hexapeptoid 11 on 2-Cholorotrityl resin (Loading 1.6 mmol/g)
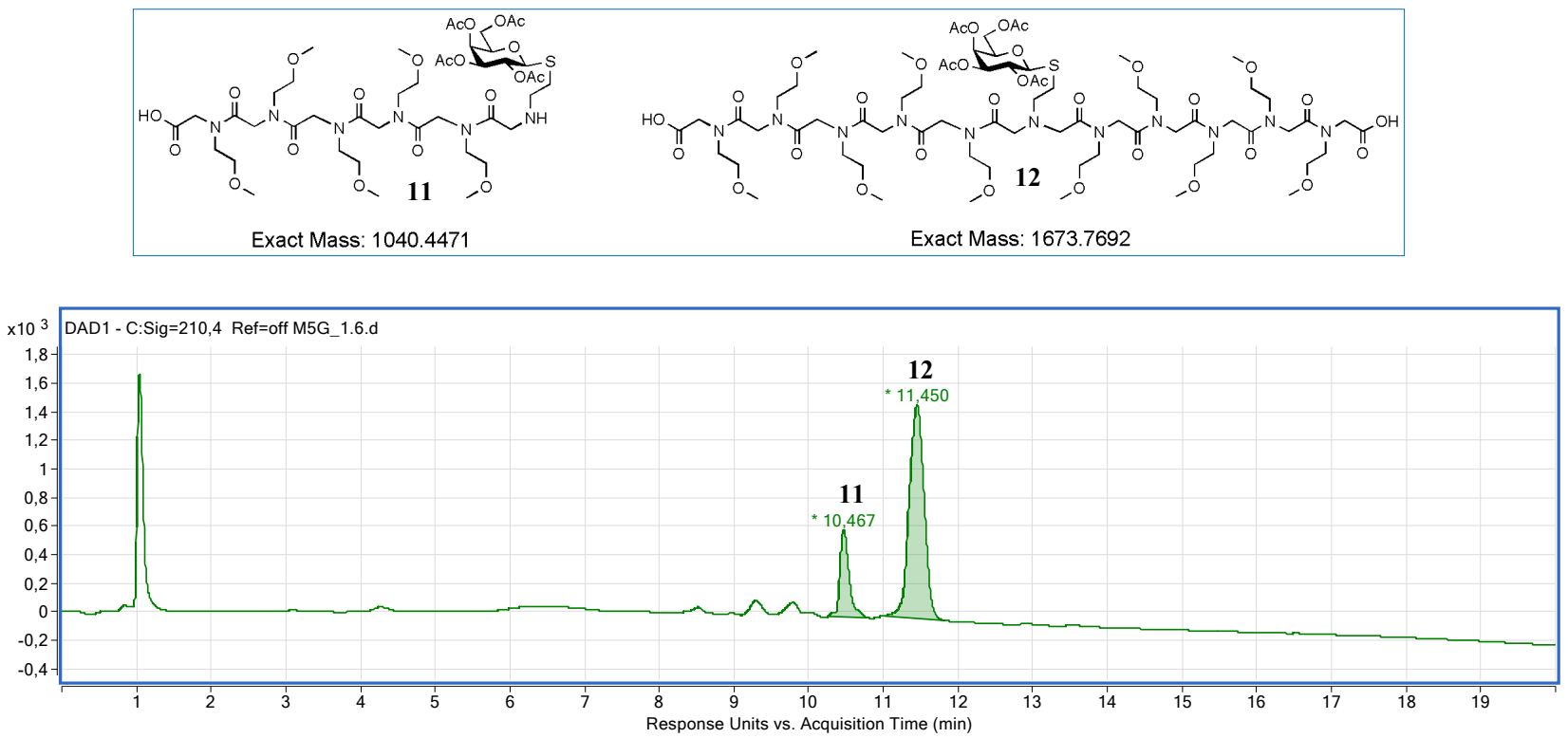

\begin{tabular}{|l|l|l|l|l|l|l|l|}
\hline Peak & MW & RT & Area & Height & Width & $\begin{array}{l}\text { Integration } \\
\text { ratio (\%) }\end{array}$ & $\begin{array}{l}\text { Real } \\
\text { ratio } \\
(\%)\end{array}$ \\
\hline $\mathbf{1 1}$ & 1041.4607 & 10.467 & 4828.2 & 592.1 & 0.52 & $19 / 81$ & $32 / 68$ \\
\hline $\mathbf{1 2}$ & 1674.7896 & 11.45 & 20575.4 & 1488.8 & 0.72 & & \\
\hline
\end{tabular}

Fig S7: Dipeptoid crudes from the submonomer synthesis by the use of different sugar moieties on 2-Chlorotrityl resin (Loading 1.0 $\mathrm{mmol} / \mathrm{g}$ )

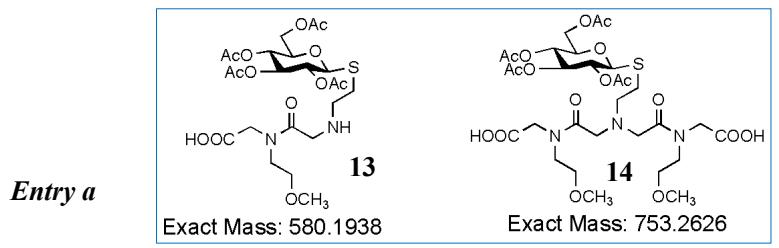




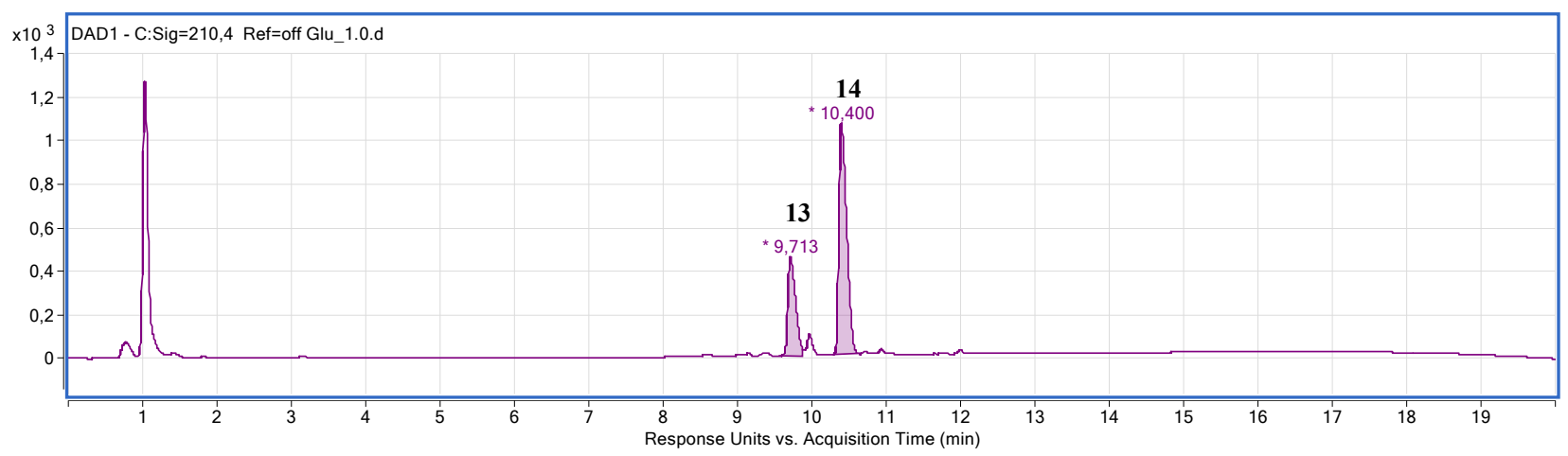

\begin{tabular}{|c|c|c|c|c|c|c|c|}
\hline Entry a & & & & & & & \\
\hline Peak & MW & RT & Area & Height & Width & $\begin{array}{l}\text { Integration } \\
\text { ratio }(\%)\end{array}$ & $\begin{array}{l}\text { Real } \\
\text { ratio } \\
(\%)\end{array}$ \\
\hline 13 & 581.2453 & 9.713 & 3091.8 & 447.0 & 0.32 & \multirow[t]{2}{*}{$28 / 72$} & \multirow[t]{2}{*}{$44 / 56$} \\
\hline 14 & 754.2676 & 10.400 & 7759.6 & 1062.0 & 0.36 & & \\
\hline
\end{tabular}
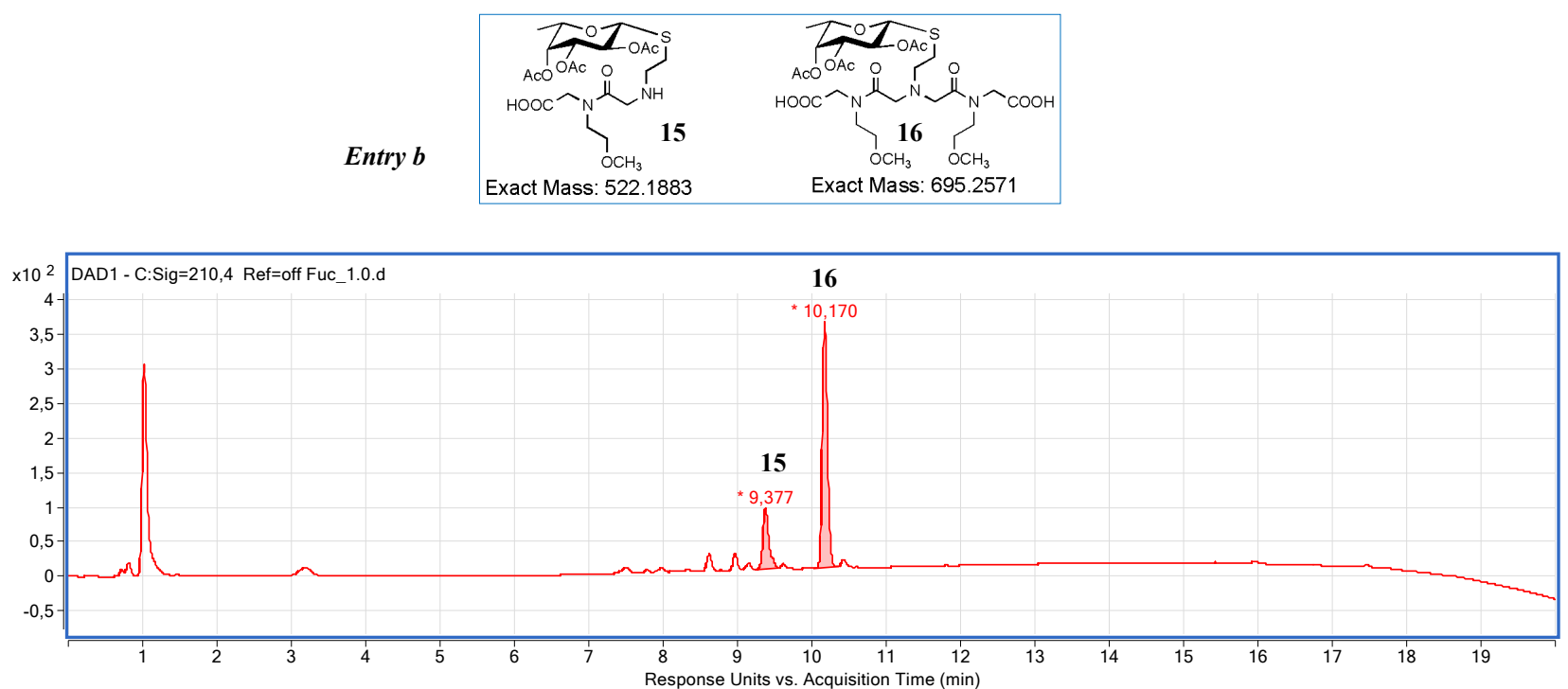

\begin{tabular}{|l|l|l|l|l|l|l|l|l|l|}
\hline Entry $\boldsymbol{b}$ & MW & & & RT & Area & Height & Width & $\begin{array}{l}\text { Integratio } \\
\text { n ratio } \\
\mathbf{\%})\end{array}$ & $\begin{array}{l}\text { Real } \\
\text { ratio } \\
(\%)\end{array}$ \\
\hline $\mathbf{1 5}$ & $\begin{array}{l}523.201 \\
8\end{array}$ & & & 9.377 & 492.2 & 88.7 & 0.29 & $22 / 78$ & $36 / 64$ \\
\cline { 1 - 2 } & $\begin{array}{l}696.272 \\
0\end{array}$ & & & 10.170 & 1736.2 & 355.7 & 0.31 & & \\
\hline
\end{tabular}

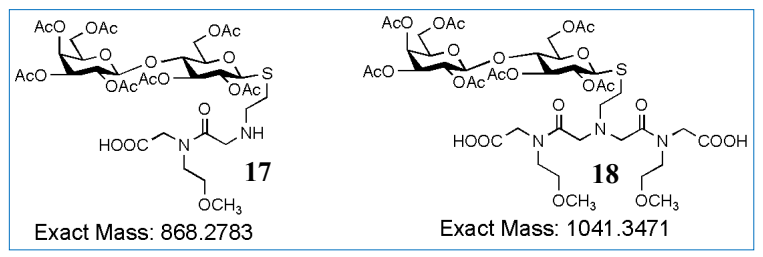




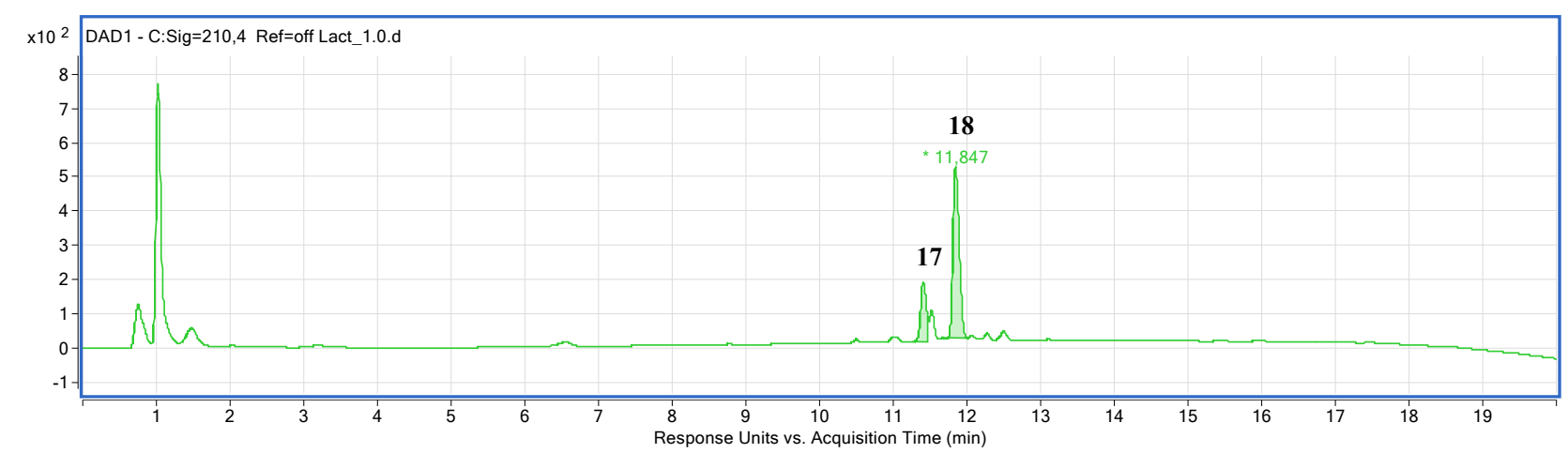

\begin{tabular}{|l|l|l|l|l|l|l|l|}
\hline Entry $\boldsymbol{c}$ & MW & RT & Area & Height & Width & $\begin{array}{l}\text { Integratio } \\
\text { n ratio } \\
(\%)\end{array}$ & $\begin{array}{l}\text { Real } \\
\text { ratio } \\
(\%)\end{array}$ \\
\hline $\mathbf{1 7}$ & 869.3094 & 11.407 & 893.2 & 172.5 & 0.19 & $23 / 77$ & $37 / 63$ \\
\hline $\mathbf{1 8}$ & 1042.383 & 11.847 & 2979.1 & 501.1 & 0.31 & & \\
\hline
\end{tabular}

Fig S8: Synthesis of Nme-N(propyl 2,3,4,6-penta-O-acetyl-1-thio-beta-D-galactosyl)glycine (Ngaltp) dipeptoid performed on 2Chlorotrityl resin (Loading $1.0 \mathrm{mmol} / \mathrm{g}$ )
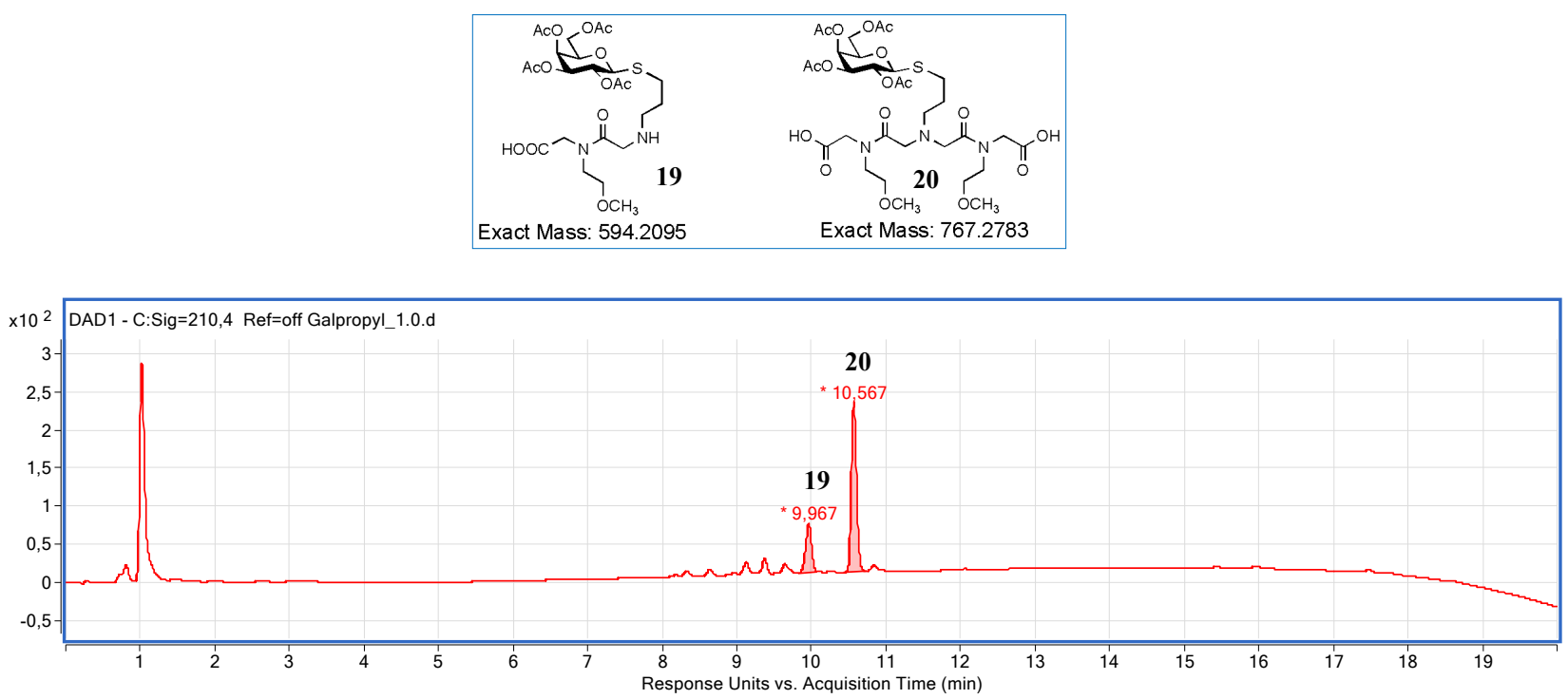

\begin{tabular}{|l|l|l|l|l|l|l|l|}
\hline Peak & MW & RT & Area & Height & Width & $\begin{array}{l}\text { Integration } \\
\text { ratio (\%) }\end{array}$ & $\begin{array}{l}\text { Real } \\
\text { ratio } \\
(\%)\end{array}$ \\
\hline $\mathbf{1 9}$ & 595.2183 & 9.967 & 353.6 & 64.6 & 0.26 & $25 / 75$ & $40 / 60$ \\
\hline $\mathbf{2 0}$ & 768.2949 & 10.567 & 1079.0 & 222.9 & 0.31 & & \\
\hline
\end{tabular}


Fig. S9: Synthesis of Nme-N(ethyl 2,3,4,6-penta-O-acetyl-1-O-beta-D-galactosyl)glycine (Ngale) dipeptoid performed on 2Chlorotrityl resin (Loading $1.0 \mathrm{mmol} / \mathrm{g}$ )
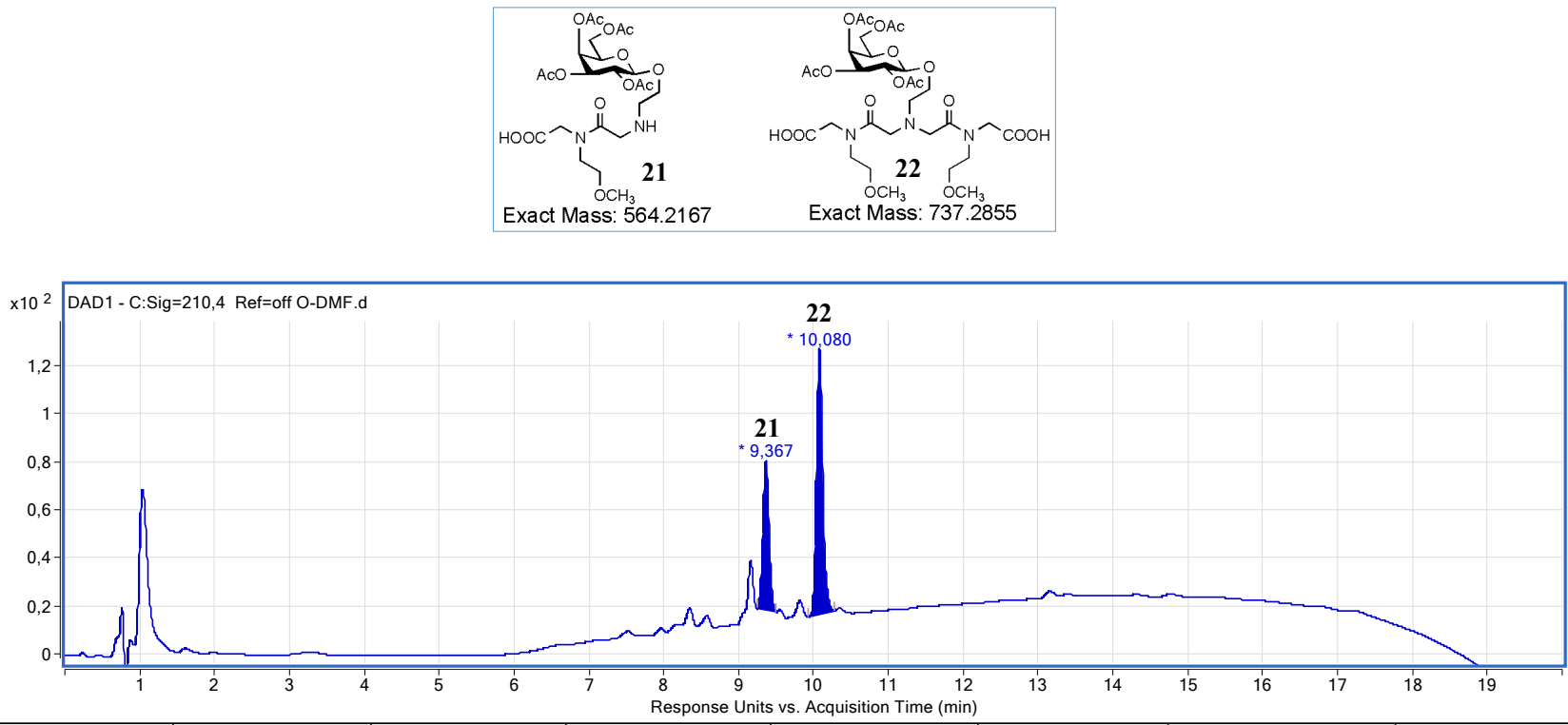

\begin{tabular}{|l|l|l|l|l|l|l|l|}
\hline Peak & MW & RT & Area & Height & Width & $\begin{array}{l}\text { Integration } \\
\text { ratio (\%) }\end{array}$ & $\begin{array}{l}\text { Real } \\
\text { ratio } \\
(\%)\end{array}$ \\
\hline $\mathbf{1 5}$ & 564.2167 & 9.367 & 356.4 & 62.4 & 0.25 & $36 / 64$ & $53 / 47$ \\
\hline $\mathbf{1 6}$ & 737.2855 & 10.08 & 637.6 & 110.48 & 0.35 & & \\
\hline
\end{tabular}

Fig. S10: Synthesis of Nme-N(ethyl 1-O-beta-D-galactosyl)glycine dipeptoid performed on 2-Chlorotrityl resin (Loading $1.0 \mathrm{mmol} / \mathrm{g}$ )
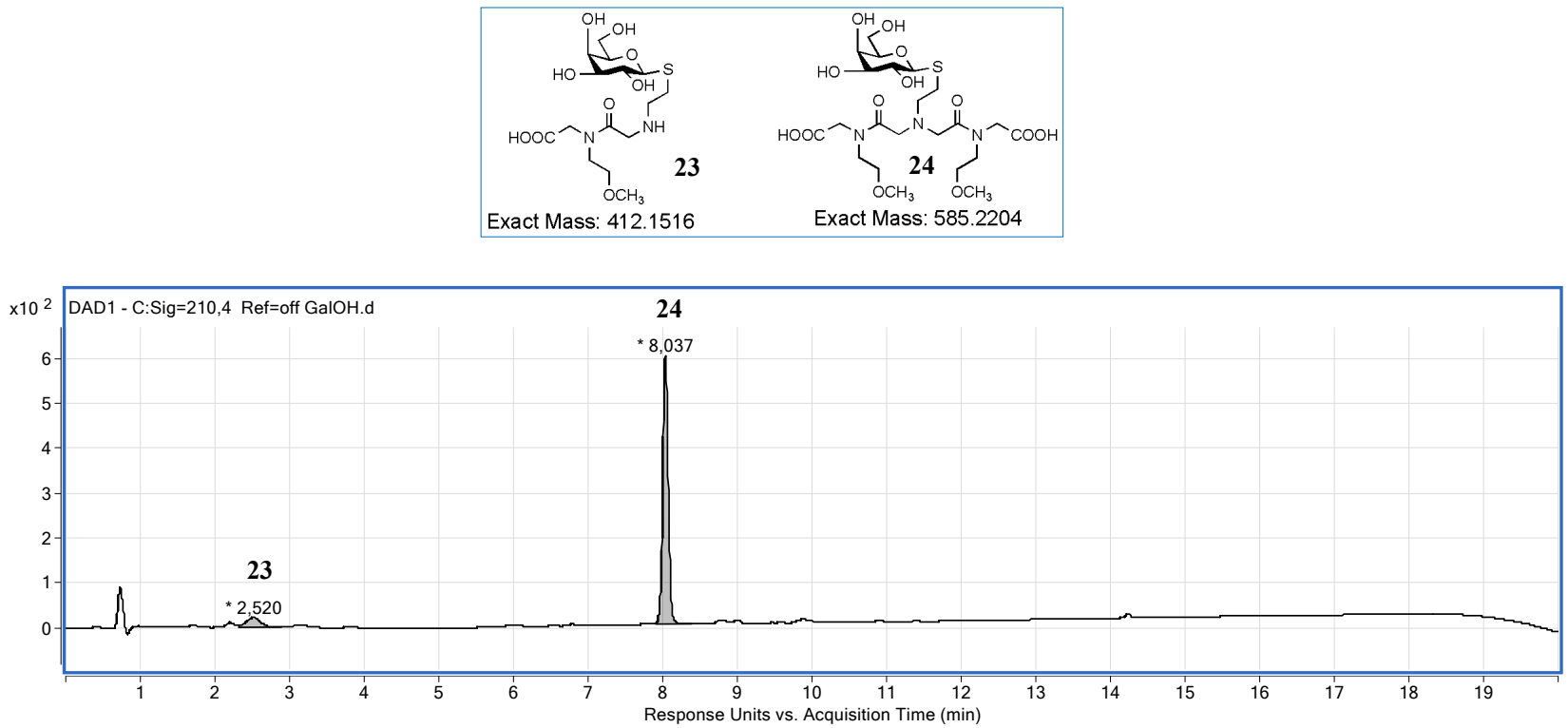

\begin{tabular}{|l|l|l|l|l|l|l|l|}
\hline Peak & MW & RT & Area & Height & Width & $\begin{array}{l}\text { Integration } \\
\text { ratio (\%) }\end{array}$ & $\begin{array}{l}\text { Real } \\
\text { ratio } \\
(\%)\end{array}$ \\
\cline { 1 - 7 } & 413.1617 & 2.520 & 289.28 & 21.32 & 0.58 & $8 / 92$ & $14 / 86$ \\
\hline $\mathbf{2 4}$ & 586.2344 & 8.037 & 3020.78 & 598.99 & 0.48 & & \\
\hline
\end{tabular}

